\title{
Toward the Standardization of Biochar Analysis: The COST Action TD1107 Interlaboratory Comparison
}

Hans Jörg Bachmann, ${ }^{\text {a }}$ Thomas D. Bucheli, ${ }^{, a}$ Alba Dieguez-Alonso, ${ }^{\mathrm{b}}$ Daniele Fabbri, ${ }^{\mathrm{c}}$ Heike Knicker, ${ }^{\mathrm{d}}$ Hans-Peter Schmidt, ${ }^{*}, \mathrm{e}$ Axel Ulbricht, ${ }^{\mathrm{f}}$ Roland Becker, ${ }^{\mathrm{g}}$ Alessandro Buscaroli, ${ }^{\mathrm{c}}$ Diane Buerge, ${ }^{\mathrm{a}}$ Andrew Cross, ${ }^{\mathrm{h}}$ Dane Dickinson, ${ }^{\mathrm{h}}$ Akio Enders, ${ }^{\mathrm{i}}$ Valdemar I. Esteves, ${ }^{\mathrm{j}}$ Michael W. H. Evangelou, ${ }^{\mathrm{k}}$ Guido Fellet, ${ }^{1}$ Kevin Friedrich, ${ }^{\mathrm{m}}$ Gabriel Gasco Guerrero, ${ }^{\mathrm{n}}$ Bruno Glaser, ${ }^{\circ}$ Ulrich M. Hanke, ${ }^{\mathrm{p}}$ Kelly Hanley, ${ }^{\mathrm{i}}$ Isabel Hilber, ${ }^{\mathrm{a}}$ Dimitrios Kalderis, ${ }^{\mathrm{q}}$ Jens Leifeld, ${ }^{\mathrm{a}}$ Ondrej Masek, ${ }^{\mathrm{h}}$ Jan Mumme, ${ }^{\mathrm{r}}$ Marina Paneque Carmona, ${ }^{d}$ Roberto Calvelo Pereira, ${ }^{\mathrm{s}}$ Frederic Rees, ${ }^{\mathrm{t}}$ Alessandro G. Rombolà, ${ }^{\mathrm{c}}$ José Maria de la Rosa, ${ }^{\mathrm{d}}$ Ruben Sakrabani, ${ }^{\mathrm{u}}$ Saran Sohi, ${ }^{\mathrm{h}}$ Gerhard Soja, ${ }^{\mathrm{v}}$ Massimo Valagussa, ${ }^{\mathrm{w}}$ Frank Verheijen, ${ }^{\mathrm{x}}$ and Franz Zehetner ${ }^{\mathrm{y}}$

${ }^{a}$ Agroscope Institute for Sustainability Sciences ISS, Reckenholzstrasse 191, 8046 Zürich, Switzerland

${ }^{\mathrm{b}}$ Technical University Berlin, Fasanenstraße 89, 10623 Berlin, Germany

${ }^{c}$ University of Bologna, CIRI EA c/o CIRSA, Via S. Alberto 163, 48123 Ravenna, Italy

${ }^{\mathrm{d}}$ Instituto de Recursos Naturales y Agrobiología de Sevilla (IRNAS-CSIC), Avenida Reina Mercedes 10, 41012 Sevilla, Spain

${ }^{\mathrm{e}}$ Ithaka Institute for Carbon Intelligence, 1974 Arbaz, Switzerland

f Eurofins Umwelt Ost GmbH, 09633 Halsbruecke OT Tuttendorf, Germany

${ }^{\mathrm{g}}$ Federal Institute for Materials Research and Testing (BAM), Richard-Willstätter-Straße 11, 12489 Berlin, Germany

${ }^{h}$ UK Biochar Research Centre, University of Edinburgh, Crew Building, Room 119, The King's Buildings, West Mains Road, Edinburgh EH9 3FF, United Kingdom

${ }^{\mathrm{i}}$ Department of Crop and Soil Sciences, Cornell University, 908 Bradfield Hall, Ithaca, New York 14853, United States

${ }^{j}$ CESAM \& Department of Chemistry, University of Aveiro, Campus de Santiago, 3810-193 Aveiro, Portugal

${ }^{\mathrm{k}}$ Institute of Terrestrial Ecosystems, ETH Zurich, Universitätstrasse 16, 8092 Zurich, Switzerland

${ }^{1}$ Department of Agricultural and Environmental Sciences, University of Udine, Via delle Scienze 208, IT-33100 Udine, Italy

${ }^{\mathrm{m}}$ Fachhochschule Bingen, Berlinstraße 109, D-55411 Bingen am Rhein, Germany

${ }^{\mathrm{n}}$ Departamento de Producción Agraria, ETSI Agrónomos, Universidad Politécnica de Madrid, Calle Ramiro de Maeztu 7, E-28040 Madrid, Spain

${ }^{\circ}$ Institut für Agrar- und Ernährungswissenschaften, Bodenbiogeochemie, Martin-Luther-Universität Halle-Wittenberg, von-Seckendorff-Platz 3, D-06120 Halle, Germany

${ }^{\mathrm{P}}$ Department of Geography, University of Zürich, Winterthurerstrasse 190, 8057 Zürich, Switzerland

${ }^{\mathrm{q}}$ Department of Environmental and Natural Resources Engineering, Technological and Educational Institute of Crete, GR-73100 Chania, Crete, Greece

${ }^{\mathrm{r}}$ Leibniz Institute for Agricultural Engineering, Potsdam-Bornim, Max-Eyth-Allee 100, D-14469 Potsdam, Germany

${ }^{s}$ Institute of Agriculture and Environment, Massey University, Private Bag 11-222, Palmerston North 4442, New Zealand

${ }^{\mathrm{t}}$ Laboratoire Sols et Environnement, 2 Avenue de la Forêt de Haye - TSA 40602, F-54518 Vandoeuvre-lès-Nancy Cedex, France

${ }^{\mathrm{u}}$ School of Energy, Environment and Agrifood, Cranfield University, Cranfield MK43 0AL, United Kingdom

${ }^{v}$ Health and Environment Department, Environmental Resources and Technologies, AIT Austrian Institute of Technology GmbH, Konrad Lorenz-Strasse 24, A-3430 Tulln, Austria

${ }^{\mathrm{w}}$ Minoprio Analisi e Certificazioni S.r.l., Viale Raimondi 54, I-22070 Vertemate con Minoprio (Como), Italy

${ }^{\mathrm{x}}$ Environment and Planning Department, CESAM - Centre for Environmental and Marine Studies, University of Aveiro, Campus Santiago, 3810-193 Aveiro, Portugal

y Institute of Soil Research, University of Natural Resources and Life Sciences (BOKU), Peter-Jordan-Strasse 82, A-1190 Vienna, Austria

Supporting Information
Received: October 19, 2015

Revised: December 17, 2015

Accepted: December 22, 2015

Published: December 22, 2015 
ABSTRACT: Biochar produced by pyrolysis of organic residues is increasingly used for soil amendment and many other applications. However, analytical methods for its physical and chemical characterization are yet far from being specifically adapted, optimized, and standardized. Therefore, COST Action TD1107 conducted an interlaboratory comparison in which 22 laboratories from 12 countries analyzed three different types of biochar for 38 physical-chemical parameters (macro- and microelements, heavy metals, polycyclic aromatic hydrocarbons, $\mathrm{pH}$, electrical conductivity, and specific surface area) with their preferential methods. The data were evaluated in detail using professional interlaboratory testing software. Whereas intralaboratory repeatability was generally good or at least acceptable, interlaboratory reproducibility was mostly not $(20 \%<$ mean reproducibility standard deviation $<460 \%$ ). This paper contributes to better comparability of biochar data published already and provides recommendations to improve and harmonize specific methods for biochar analysis in the future.

KEYWORDS: biochar analysis, biochar certification, round-robin test, interlaboratory test, charcoal, polycyclic aromatic hydrocarbons, heavy metals

\section{INTRODUCTION}

Biochar is a heterogeneous substance rich in aromatic carbon (C) and minerals, which is produced by pyrolysis of sustainably obtained biomass. ${ }^{1}$ Multiple uses of biochar in various areas such as crop production, animal farming, wastewater treatment, in building materials, or in the textile industry are all based on specific chemical-physical properties of the biochar material. It is therefore fundamental for the comparability of scientific results, for regulators, as well as for future industrial implementations, to develop analytical standards, which eventually become the base of biochar certification and classification systems. ${ }^{2}$ When biochar developed into a topic of research in fields such as soil science or plant nutrition during the past 5-10 years, awareness and understanding of the very peculiar material properties of biochar were low. Instead of applying and further developing analytical methods specially adapted for charcoal, most laboratories and biochar researchers used-and still use-methods originally established for soils, fertilizers, and composts.

As collaboration was sparse until recently, biochar analysis evolved rather discordantly, which aggravated comparability of results from different laboratories and publications. To address the state-of-the-art in biochar analysis and to eventually develop standard analytical methods for biochar characterization and certification, in 2013 EU-COST Action TD1107 "Biochar as option for sustainable resource management" organized an interlaboratory comparison. Although such exercises had been performed in the past for matrices such as coal, ${ }^{3,4}$ carbonaceous aerosols, ${ }^{5,6}$ and source rock, ${ }^{7}$ to our knowledge, the present study is the first of its kind for biochar. Interested biochar research groups were invited to characterize three different biochars by analytical techniques they considered to be most appropriate.

The three biochars were produced from different feedstocks with the same pyrolysis technology under comparable processing conditions, although in three different facilities. Representative subsamples of these biochars were sent to 22 participating laboratories in 12 countries. The objective of the comparison was not to evaluate the interlaboratory reproducibility using the same standard methods but to estimate the reliability of analytical results obtained by laboratories using their habitual methods of biochar analyses; hence, no default methods were stipulated. A standard set of characterizing parameters was selected along with some optional extended parameters that are especially helpful to understand the biochar functionality. The resulting data of the 38 parameters were subjected to statistical evaluations to check the variability between the used methods and to compare the respective reliabilities. To verify the performance of the methods recommended by the European Biochar Certificate (EBC), data from participating laboratories were compared with the results of Eurofins Laboratories, that is, the only laboratory which consistently followed them.

The objectives of this exercise were (1) to estimate the reliability of widely used analytical methods for biochar, (2) to facilitate comparability of biochar data in the scientific literature, and (3) to provide recommendations for methods suitable for biochar quality assurance and control and certification.

\section{MATERIALS AND METHODS}

Feedstock and Preparation of the Biochars. Three different feedstocks, namely, shavings from wood chip production, a blend of paper sludge and wheat husks, and sewage sludge (at $75 \%$ dry weight $(\mathrm{dw})$ ), were pyrolyzed with PYREG 500 - III pyrolysis units (PYREG GmbH, Dörth, Germany) by Swiss Biochar (Lausanne, Switzerland), Sonnenerde $\mathrm{GmbH}$ (Riedlingsdorf, Austria), and PYREG GmbH to produce biochars $\mathrm{BC} 1, \mathrm{BC} 2$, and $\mathrm{BC} 3$, respectively. Pyrolysis took place for $20 \mathrm{~min}$ at maximum temperatures of 620 (BC1), 500 (BC2), and $600{ }^{\circ} \mathrm{C}(\mathrm{BC} 3)$. No inert gas was used as flush gas to drive off pyrolytic vapors. The biochars were allowed to gas out for $5 \mathrm{~min}$ and were quenched with water to $30 \%$ water content. The materials were stored under ambient air for several days and then filled into bags of $1.5 \mathrm{~m}^{3}$. Before the aliquots (2000 g per biochar sample) were gathered for distribution to the participating laboratories, each of the three biochar lots was homogenized following the sampling method proposed by Bucheli et al. ${ }^{9}$ In short, each pile was shoveled three times from one place to another. The size was then reduced by removing $80 \mathrm{~kg}$ from the total lot; this $80 \mathrm{~kg}$ portion was again shoveled three times from one place to another. This procedure allowed for the generation of subsamples that were representative of the total lot. ${ }^{9}$ After receiving the samples, the participants were asked to dry them at $40{ }^{\circ} \mathrm{C}$ and to store them airtight below $5{ }^{\circ} \mathrm{C}$. Consequently, all results refer to a dw basis, unless otherwise stated.

Participants and Analytical Methods. Overall, 22 laboratories were participating in the interlaboratory comparison, each contributing a data set with results for one or more parameters. Laboratories were anonymized and numbered from 1 to 24 (LAB_01-LAB_24, without LAB_03 and LAB_14, which delivered data for parameters not included in this study). In cases when laboratories used different sample preparations or methods to analyze the same parameter, an A, $\mathrm{B}$, or $\mathrm{C}$ was added to the laboratory code to differentiate between the respective results (e.g., LAB_07A). Biochar parameters and analytes of primary interest were selected as those stipulated by the International Biochar Initiative (IBI) ${ }^{10}$ and the $\mathrm{EBC},{ }^{1}$ but the participants were free to analyze only part of them or to report additional ones, depending on their analytical capabilities. Participants were also asked to provide detailed protocols of their sample preparation and analytical methods. Table S1 (in the Supporting Information) compiles the methods used by the different laboratories to analyze the parameters. The EBC and IBI recommended methods are described elsewhere. ${ }^{1,8,10}$ 
Data Evaluation. All data were treated with PROLab Plus (version 2015.11.3.0) software from QuoData GmbH (Dresden, Germany) for interlaboratory testing. The fundamental evaluation was performed according to DIN 38402 A45. ${ }^{11}$ The software delivers a comprehensive set of statistical key data for the interpretation of interlaboratory comparison results. As consensus values, the robust means were calculated according to the method of Hampel et al., ${ }^{12}$ and robust standard deviations (SD) were found by Q-estimation. ${ }^{13}$ The SD was taken as a measure for the reproducibility of the analytical results between the laboratories (reproducibility SD). The advantage of using robust estimation methods is that values lying far from the majority of results do not have to be excluded as so-called outliers because their influence is reduced in an appropriate manner. The exclusion of outliers bears the risk of an overly optimistic estimation of the SD, especially in the case of an exploratory interlaboratory comparison such as this one. The exclusion of outliers would further affect the values for the relative $\mathrm{SD}$ (ratio of $\mathrm{SD}$ to the mean) and the $\mathrm{Zu}$ scores, ${ }^{11}$ which are calculated as difference in reproducibility SD between the consensus values and the laboratory results, with compensation for the right-skewness of their distribution.

Besides the already mentioned parameters, we used the concentration-dependent values of the Horwitz function. ${ }^{14}$ They are an empirical and independent reference for the SD that can be expected in an established round-robin test under optimal conditions. In this case, the so-called Horwitz ratio (HorRat), ${ }^{15}$ that is, the ratio of the $\mathrm{SD}$ of the mean concentration divided by the Horwitz value, lies between 0.5 and 2. As the Horwitz function is defined for concentrations, there are no Horwitz values for $\mathrm{pH}$, electrical conductivity (EC), and specific surface area (SSA).

In case a laboratory reported multiple results for a given parameter, the robust mean was used as laboratory value and the robust SD of this mean as a measure of the repeatability of the analysis within this laboratory (repeatability SD). For comparison, a reference value was defined for each parameter, which was based on currently recommended
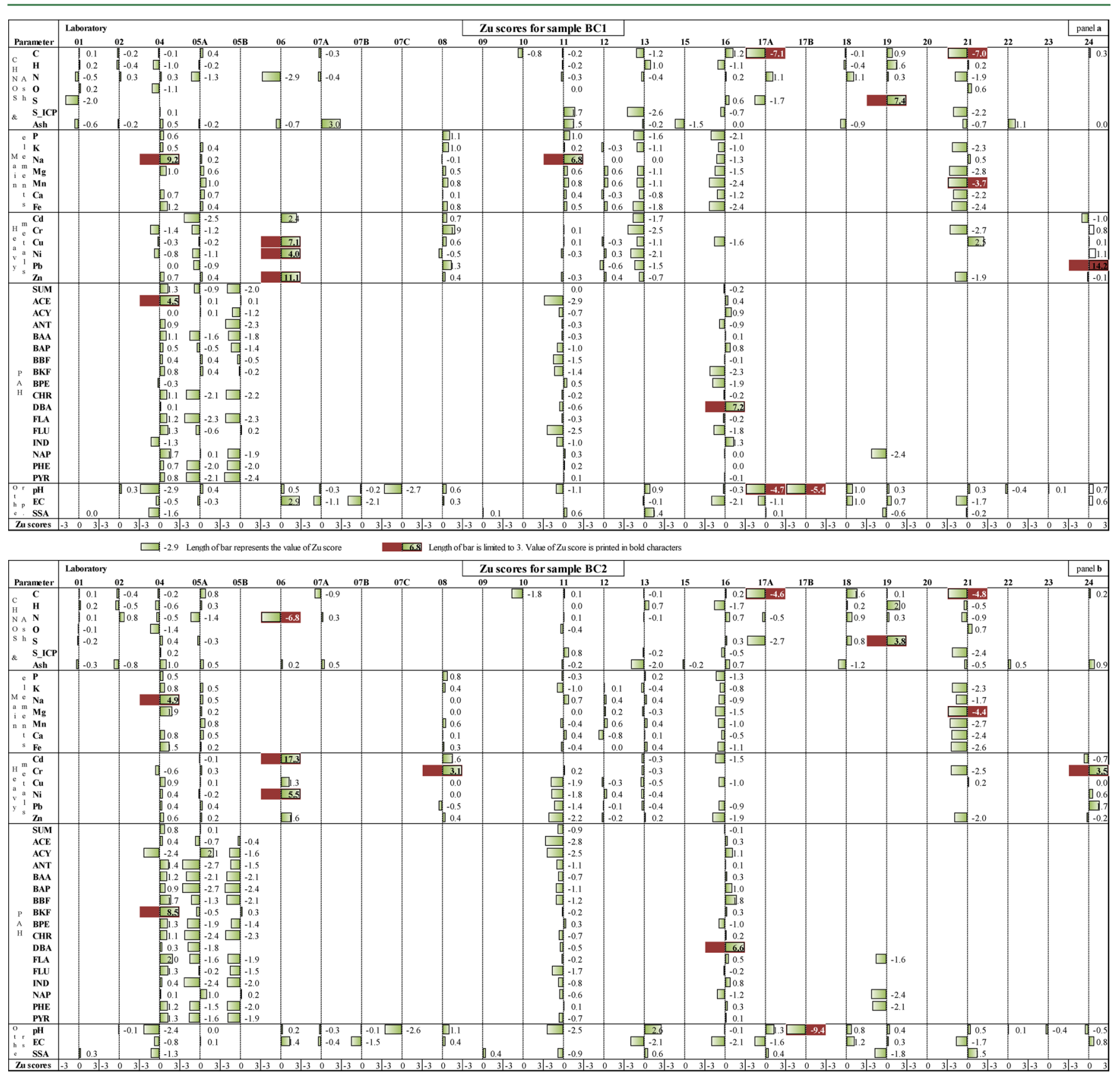

$\square-2.9$ Lenoth of bar represents the value of $Z$ u score

6.8 Lensth of bar is limited to 3 . Value of Zu score is printed in bold characters

Figure 1. continued 


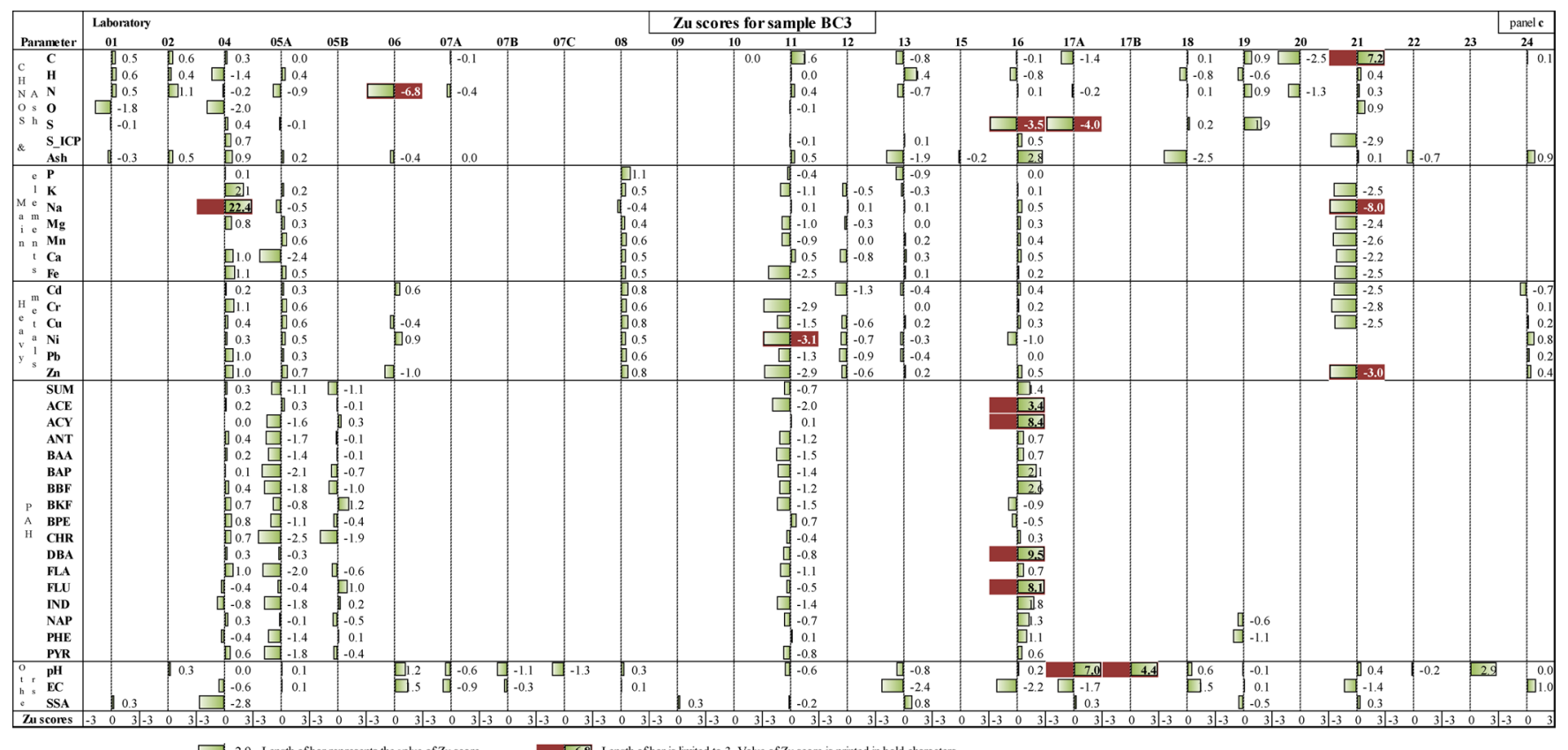

Figure 1. Charts of $\mathrm{Zu}$ scores (green bars) for all parameters, laboratories, and biochars (BC1 (a), BC2 (b), and BC3 (c)). Results with $\mid \mathrm{Zu}$ scoresl $>3$ (red bars) are generally considered as outliers that have to be specifically examined.

methods for biochar analysis by the EBC. ${ }^{8}$ All reference values were generated in the same laboratory (LAB_04).

We used $\mathrm{Zu}$ score plots for a general overview of the data set (Figure 1), as well as box plots (see Figure 2a and Figure S1) and Kernel density plots (see Figure $2 \mathrm{~b}$ and Figure S2) for visualization of laboratory results of each parameter. The limits of tolerance are defined as $\pm 3 \mathrm{Zu}$ scores. The box plot gives a detailed overview of the results of just one parameter and allows direct comparison of the reproducibility SD and the repeatability SD. A ratio of these values $>3$ strongly indicates systematic differences between laboratory results. The Kernel density plot gives a much better picture of the distribution of the laboratory values than the box plot. It often allows a clear distinction between different methods used. If the main modus comprises $<75 \%$ and/or differs significantly from the mean, then it is doubtful that all results are comparable. This indication of systematic differences is much stronger than the ones mentioned above.

\section{RESULTS AND DISCUSSION}

In the following, the results will be grouped according to the main parameters and discussed individually in subsections. First, some general remarks on the results of the evaluation of the interlaboratory comparison are given. Figure 1 presents the $\mathrm{Zu}$ scores for all parameters, laboratories, and all three biochars (panels a-c). It serves as an overview of all results of the interlaboratory comparison. Laboratory results with $\mathrm{IZu}$ scoresl $\leq 2$ lie within the majority of results and are generally considered as inconspicuous. Those with $2<\mid \mathrm{Zu}$ scores $\mid \leq 3$ are farther deviating and attract more attention, but are, in the case of a first exploratory interlaboratory comparison, still in a usual range. Results with $\mid \mathrm{Zu}$ scores $\mid>3$ are generally considered as outliers that have to be specifically examined. In a methodological interlaboratory comparison as in this study, they are strong indicators for systematic differences in the results due to the use of unequal analytical methods or variability in the application of the same method. The $\mathrm{Zu}$ scores plot can also be used to recognize general tendencies, for example, that most of the results of a laboratory are located systematically above or below the consensus values. However,
$\mathrm{Zu}$ scores represent only a relative scale that can be used to detect outliers and general tendencies, provided that the reproducibility of results between laboratories is not too low. For a more robust assessment of the analytical quality of the results, the relative reproducibility $\mathrm{SD}$ between the laboratories as well as the HorRat have to be used.

Consequently, Table 1 shows the statistical key data for each parameter resulting from the evaluation of the interlaboratory comparison. They are grouped in the same manner as in the following subsections. Generally, it is obvious that the relative reproducibility SD values varied broadly and were in most cases quite high, whereas the relative repeatability SD values were much closer and in most cases within the expected range around the Horwitz value. In contrast, most of the HorRat values for the reproducibility SD were far above 2 . This clearly indicates that the between-laboratory reproducibility is currently not satisfactory for the majority of the parameters and has to be improved.

C, H, N, O, S, and Ash Analysis. Total Carbon (C). During the pyrolysis of an organic feedstock, the majority of the organic matter (OM) is released as syngas and pyrolytic oils, leading to a relative enrichment of ash. However, the final concentration and composition of OM vary with feedstock and pyrolysis conditions. ${ }^{16}$ Because it largely affects the properties of the char, the organic carbon $\left(\mathrm{C}_{\mathrm{org}}\right)$ content is commonly used as an important parameter to identify biochar. Accordingly, chars with a $\mathrm{C}_{\text {org }}$ content $<50 \%$ of their $\mathrm{dw}$ are not considered biochars but are classified as pyrogenic carbonaceous material (PCM) by the $\mathrm{EBC}^{1}$ or lower classed biochars by the IBI. ${ }^{10}$

In the present interlaboratory comparison, almost all participating laboratories used elemental analysis via combustion $>950{ }^{\circ} \mathrm{C}$ for the determination of the $\mathrm{C}$ content (Table S1). Here it has to be borne in mind that with this method, the total carbon (inorganic and $\mathrm{C}_{\text {org }}$ ) is determined. If carbonates are not present, it can be assumed that the analyzed total $\mathrm{C}$ represents $\mathrm{C}_{\text {org }}$. However, if carbonates are present, as in $\mathrm{BC} 2$, the proportion 

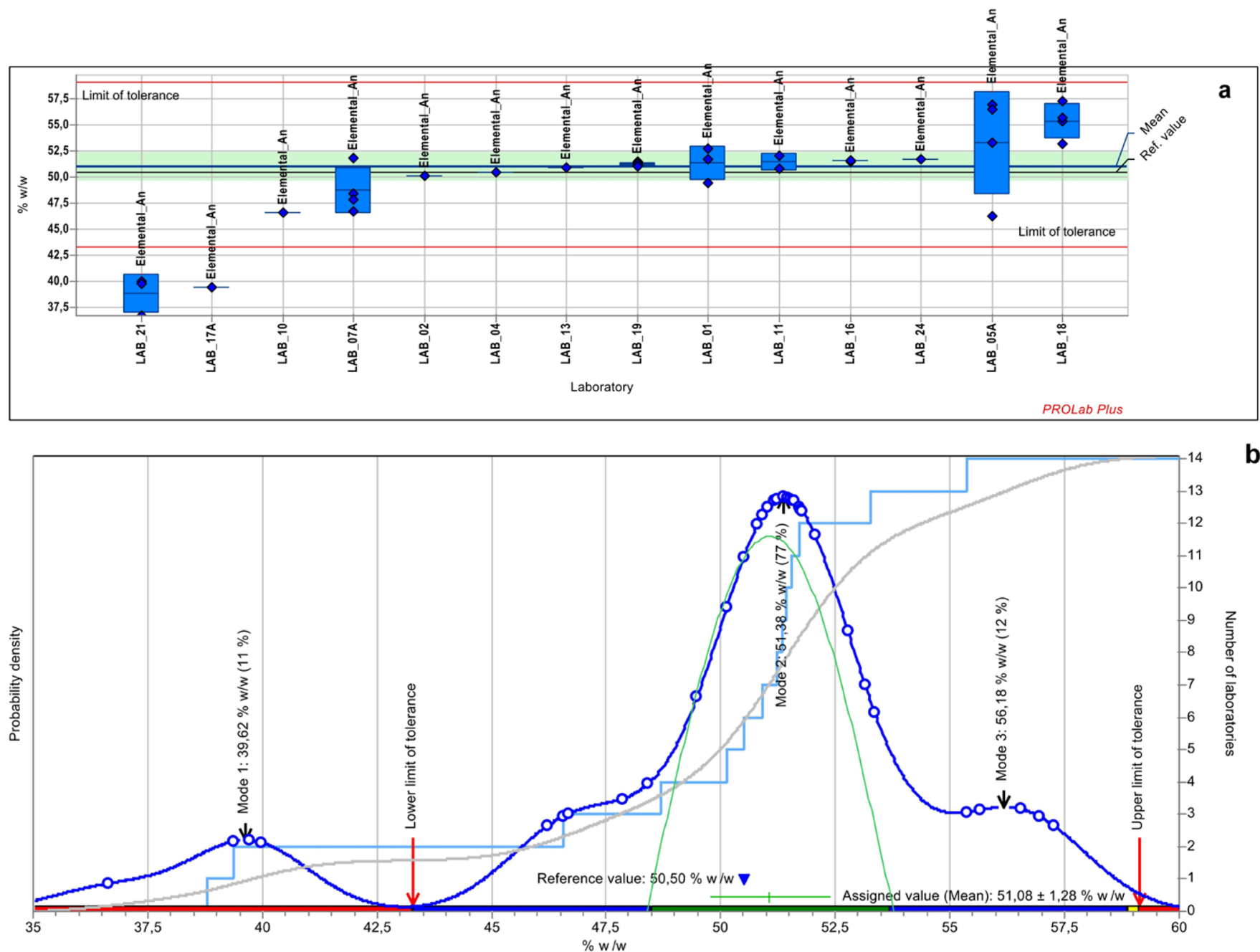

Figure 2. Examples of a box plot (a) and a Kernel density plot (b) for the parameter C (\% w/w) in BC2. The box plot (a) graphically presents all laboratory values for one given parameter in one sample. On the abscissa the laboratories are stringed in ascending order of the mean of their results. The ordinate is the scale for the values of the parameter analyzed, here $\% \mathrm{w} / \mathrm{w}$ for the $\mathrm{C}$ content. The individual laboratory results are depicted as blue rhombuses with a bar for the mean or the single value. In the case of multiple results, the box comprises the middle 50\% quantile (i.e., the $25-$ $75 \%$ percentile range). Above the box, codes for the analytical method and sample preparation used by the laboratory are specified. A complete compilation of all methods, preparations, and codes is presented in Table S1 in the Supporting Information. Two broad horizontal lines with legends on the right side mark the positions of the robust mean as consensus value (blue) and the reference value (black) obtained by LAB_04 using strictly the methods actually recommended by the $\mathrm{EBC},{ }^{8}$ respectively. The limits of tolerance (red lines) are defined as $\pm 3 \mathrm{Zu}$ scores. Values outside these limits can be considered as significantly different from the majority of results. In the Kernel density plot (b), the abscissa is the scale for the laboratory results, here in $\% \mathrm{w} / \mathrm{w}$ for the $\mathrm{C}$ content of $\mathrm{BC} 2$. The values for the mean, the reference values, and the limits of tolerance refer to the aforementioned scale and have the same meaning as in the box plots. The left ordinate is a probability scale for the Kernel density, whereas the right ordinate represents the number of laboratories. The blue line represents the Kernel density distribution calculated from all laboratory results, which are pictured by circles. At the maxima of the Kernel density, the parameter values and weights of the corresponding modi were specified. The green line illustrates the normal distribution. The gray curve represents the cumulated probability and the step curve (light blue) the cumulated number of laboratories. A complete compilation of box plots and Kernel density plots is included in the Supporting Information (Figures S1 and S2).

of $\mathrm{C}_{\text {org }}$ has to be determined either by removal of inorganic $\mathrm{C}$ with acid or by removal of $\mathrm{OM}$ via muffling at $500{ }^{\circ} \mathrm{C}$. Unfortunately, only six laboratories reported values for $\mathrm{C}_{\text {org. }}$. The results were quite scattered and partly inconsistent (e.g., $\mathrm{C}_{\text {org }}>\mathrm{TC}$ ) so that a well-founded interlaboratory evaluation was impossible.

With the exception of values obtained from two laboratories ( $L A B \_17 A$ and $L A B \quad 21$ ), the $\mathrm{Zu}$ scores for the $\mathrm{C}$ contents were $<|3|$ (Figure 1) and thus within the limits of tolerance (Figure S1, pp 5, 45, 85). The mean C content of $81.9 \%$ of BC1 (Table 1) was close to the reference value $(81.2 \%)$, which allows an unambiguous classification as biochar. For BC2 a mean C content of $51.1 \%$ was calculated (Table 1). With a mean C content of $18.5 \%$ (Table 1), BC3 did not fulfill the criteria of an EBC-certified biochar and would have to be considered as PCM or as a class 3 biochar following the IBI standard. ${ }^{10}$

A bimodal pattern with $89 \%$ mode 2 representation $(81.8 \%$ C) and $11 \%$ mode 1 contribution $(45.5 \% \mathrm{C})$ was observed from the Kernel density plot of BC1 (Figure S2, p 125). BC2 plots trimodal with modes at $39.6 \% \mathrm{C}(11 \%), 51.4 \% \mathrm{C}(77 \%)$, and $56.2 \% \mathrm{C}(12 \%)$ (Figure S2, p 165). Possibly, the hygroscopic properties observed for these samples contributed to this multimodal distribution. Hygroscopic substances tend to ab- or adsorb water from their surroundings, which increases their weight. If such samples are not carefully dried before preparation for elemental analysis, $\mathrm{C}$ contents tend to be too low. 
Table 1. Statistical Evaluation ${ }^{\mathrm{a}}$ of the Analytical Results Reported by the Participants of the Biochar Interlaboratory Comparison

\begin{tabular}{|c|c|c|c|c|c|c|c|c|c|c|c|c|c|}
\hline \multirow{4}{*}{$\begin{array}{l}\text { Group } \\
\text { CHNOS } \\
\text { \& Ash }\end{array}$} & \multirow{3}{*}{$\frac{\begin{array}{c}\text { Para- } \\
\text { meter }\end{array}}{\underline{\underline{\mathrm{C}}}}$} & \multirow{2}{*}{ Sample } & \multirow[t]{2}{*}{ Unit } & \multirow[t]{2}{*}{ Mean value } & Reference & & er of & Reprodu & ility SD & Horwitz f & ction & Repeat & ility SD \\
\hline & & & & & value & labs & results & absolute & relative & value & ratio & absolute & relative \\
\hline & & $\mathrm{BC} 1$ & $\%$ & 81.9 & 81.2 & 14 & 35 & 5.2 & $6.4 \%$ & 1.7 & 3.1 & 2.0 & $2.4 \%$ \\
\hline & & $\mathrm{BC} 2$ & $\%$ & 51.1 & 50.5 & 14 & 35 & 2.6 & $5.1 \%$ & 1.1 & 2.3 & 1.4 & $2.8 \%$ \\
\hline & & BC3 & $\%$ & 18.5 & 18.7 & 15 & 37 & 0.7 & $3.9 \%$ & 0.5 & $\underline{1.5}$ & 0.1 & $0.6 \%$ \\
\hline & $\underline{\mathbf{H}}$ & $\mathrm{BC} 1$ & $\%$ & 1.48 & 1.19 & 10 & 24 & 0.31 & $21.0 \%$ & 0.06 & 5.5 & 0.12 & $8.2 \%$ \\
\hline & & $\mathrm{BC} 2$ & $\%$ & 1.73 & 1.55 & 10 & 28 & 0.31 & $17.7 \%$ & 0.06 & 4.8 & 0.05 & $2.8 \%$ \\
\hline & & $\mathrm{BC} 3$ & $\%$ & 1.18 & 0.93 & 10 & 29 & 0.18 & $15.4 \%$ & 0.05 & 3.9 & 0.03 & $2.7 \%$ \\
\hline & $\mathrm{N}$ & $\mathrm{BC} 1$ & $\%$ & 0.35 & 0.42 & 13 & 36 & 0.21 & $59.3 \%$ & 0.02 & 12.4 & 0.02 & $5.6 \%$ \\
\hline & & $\mathrm{BC} 2$ & $\%$ & 1.39 & 1.29 & 13 & 36 & 0.20 & $14.4 \%$ & 0.05 & 3.8 & 0.03 & $2.2 \%$ \\
\hline & & $\mathrm{BC} 3$ & $\%$ & 2.13 & 2.10 & 13 & 38 & 0.16 & $7.5 \%$ & 0.08 & 2.1 & 0.04 & $1.9 \%$ \\
\hline & $O$ & BC1 & $\%$ & 5.8 & 3.8 & 4 & 9 & 2.0 & $35.3 \%$ & 0.2 & 11.5 & 0.2 & $2.9 \%$ \\
\hline & & $\mathrm{BC} 2$ & $\%$ & 12.1 & 6.4 & 4 & 9 & 8.6 & $71.5 \%$ & 0.3 & 26.0 & 1.2 & $9.9 \%$ \\
\hline & & $\mathrm{BC} 3$ & $\%$ & 10.5 & 3.7 & 4 & 9 & 12.5 & $119.2 \%$ & 0.3 & 42.4 & 0.8 & $7.9 \%$ \\
\hline & $S$ & $\mathrm{BC} 1$ & $\%$ & 0.042 & - & 4 & 13 & 0.067 & $160.6 \%$ & 0.003 & 22.3 & 0.067 & $160.6 \%$ \\
\hline & & $\mathrm{BC} 2$ & $\%$ & 0.116 & 0.140 & 7 & 22 & 0.045 & $38.9 \%$ & 0.006 & 7.5 & 0.019 & $16.7 \%$ \\
\hline & & $\mathrm{BC} 3$ & $\%$ & 0.691 & 0.760 & 7 & 23 & 0.170 & $24.6 \%$ & 0.029 & 5.9 & 0.027 & $3.9 \%$ \\
\hline & $S I C P-$ & $\mathrm{BC} 1$ & $\%$ & 0.02 & 0.02 & 5 & 10 & 0.02 & $112.2 \%$ & 0.00 & 21.0 & 0.00 & $6.9 \%$ \\
\hline & OES & $\mathrm{BC} 2$ & $\%$ & 0.13 & 0.16 & 5 & 10 & 0.11 & $84.9 \%$ & 0.01 & 15.6 & 0.00 & $3.0 \%$ \\
\hline & & $\mathrm{BC} 3$ & $\%$ & 0.58 & 0.72 & 5 & 10 & 0.17 & $29.3 \%$ & 0.03 & 6.8 & 0.01 & $1.8 \%$ \\
\hline & $\underline{\text { Ash }}$ & $\mathrm{BC} 1$ & $\%$ & 11.35 & 13.30 & 14 & 25 & 3.58 & $31.5 \%$ & 0.31 & 11.7 & 1.04 & $9.5 \%$ \\
\hline & & $\mathrm{BC} 2$ & $\%$ & 34.78 & 40.20 & 14 & 26 & 4.98 & $14.3 \%$ & 0.82 & 6.1 & 0.30 & $0.9 \%$ \\
\hline & & $\mathrm{BC} 3$ & $\%$ & 72.45 & 73.80 & 14 & 25 & 1.54 & $2.1 \%$ & 1.52 & $\underline{1.0}$ & 0.20 & $0.3 \%$ \\
\hline Main & $\underline{\mathbf{P}}$ & BC1 & $\mathrm{mg} / \mathrm{kg}$ & 765 & 870 & 5 & 11 & 152 & $19.8 \%$ & 45 & 3.4 & 3 & $0.4 \%$ \\
\hline elements & & $\mathrm{BC} 2$ & $\mathrm{mg} / \mathrm{kg}$ & 6'054 & $6^{\prime} 610$ & 5 & 11 & 1'049 & $17.3 \%$ & 261 & 4.0 & 59 & $1.0 \%$ \\
\hline & & $\mathrm{BC} 3$ & $\mathrm{mg} / \mathrm{kg}$ & $60^{\prime} 531$ & $61^{\prime} 500$ & 5 & 11 & $9^{\prime} 894$ & $16.3 \%$ & 1'846 & 5.4 & 727 & $1.2 \%$ \\
\hline & $K$ & $\mathrm{BC} 1$ & $\mathrm{mg} / \mathrm{kg}$ & $6 ' 763$ & $9^{\prime} 400$ & 8 & 19 & 4'144 & $61.3 \%$ & 287 & 14.4 & 111 & $1.6 \%$ \\
\hline & & $\mathrm{BC} 2$ & $\mathrm{mg} / \mathrm{kg}$ & $10^{\prime} 016$ & $16^{\prime} 800$ & 8 & 19 & $6^{\prime} 869$ & $68.6 \%$ & 401 & 17.1 & 183 & $1.8 \%$ \\
\hline & & $\mathrm{BC} 3$ & $\mathrm{mg} / \mathrm{kg}$ & 4'096 & $10^{\prime} 300$ & 8 & 19 & $2^{\prime} 416$ & $59.0 \%$ & 187 & 12.9 & 54 & $1.3 \%$ \\
\hline & $\mathrm{Na}$ & $\mathrm{BC} 1$ & $\mathrm{mg} / \mathrm{kg}$ & 103 & 690 & 8 & 19 & 52 & $50.5 \%$ & 8 & 6.3 & 8 & $7.8 \%$ \\
\hline & & $\mathrm{BC} 2$ & $\mathrm{mg} / \mathrm{kg}$ & 308 & 910 & 8 & 19 & 102 & $33.0 \%$ & 21 & 4.9 & 11 & $3.4 \%$ \\
\hline & & $\mathrm{BC} 3$ & $\mathrm{mg} / \mathrm{kg}$ & $1 ' 273$ & 4'140 & 8 & 19 & 123 & $9.7 \%$ & 69 & $\underline{1.8}$ & 26 & $2.1 \%$ \\
\hline & $\mathrm{Mg}$ & $\mathrm{BC} 1$ & $\mathrm{mg} / \mathrm{kg}$ & $1 ' 724$ & $2^{\prime} 320$ & 8 & 19 & 489 & $28.4 \%$ & 90 & 5.4 & 25 & $1.5 \%$ \\
\hline & & $\mathrm{BC} 2$ & $\mathrm{mg} / \mathrm{kg}$ & $3^{\prime} 234$ & $4^{\prime} 530$ & 8 & 19 & 614 & $19.0 \%$ & 153 & 4.0 & 124 & $3.8 \%$ \\
\hline & & $\mathrm{BC} 3$ & $\mathrm{mg} / \mathrm{kg}$ & $8^{\prime} 877$ & $13^{\prime} 300$ & 8 & 19 & 4'543 & $51.2 \%$ & 361 & 12.6 & 115 & $1.3 \%$ \\
\hline & $\mathrm{Mn}$ & $\mathrm{BC} 1$ & $\mathrm{mg} / \mathrm{kg}$ & 301 & - & 7 & 18 & 66 & $22.0 \%$ & 20 & 3.2 & 5 & $1.5 \%$ \\
\hline & & $\mathrm{BC} 2$ & $\mathrm{mg} / \mathrm{kg}$ & 127 & - & 7 & 18 & 41 & $32.2 \%$ & 10 & 4.2 & 5 & $3.7 \%$ \\
\hline & & $\mathrm{BC} 3$ & $\mathrm{mg} / \mathrm{kg}$ & 514 & - & 7 & 18 & 224 & $43.6 \%$ & 32 & 7.0 & 10 & $1.9 \%$ \\
\hline & $\mathrm{Ca}$ & $\mathrm{BC} 1$ & $\mathrm{mg} / \mathrm{kg}$ & $21^{\prime} 705$ & $33^{\prime} 700$ & 8 & 19 & $14^{\prime} 083$ & $64.9 \%$ & 773 & 18.2 & 629 & $2.9 \%$ \\
\hline & & $\mathrm{BC} 2$ & $\mathrm{mg} / \mathrm{kg}$ & $62 ' 219$ & $89^{\prime} 200$ & 8 & 19 & $27 ' 741$ & $44.6 \%$ & 1'890 & 14.7 & $2^{\prime} 775$ & $4.5 \%$ \\
\hline & & $\mathrm{BC} 3$ & $\mathrm{mg} / \mathrm{kg}$ & $33^{\prime} 194$ & $54^{\prime} 400$ & 8 & 19 & 17316 & $52.2 \%$ & 1'108 & 15.6 & 334 & $1.0 \%$ \\
\hline & $\mathrm{Fe}$ & BC1 & $\mathrm{mg} / \mathrm{kg}$ & $1^{\prime} 087$ & $1^{\prime} 840$ & 8 & 19 & 492 & $45.3 \%$ & 61 & 8.1 & 38 & $3.5 \%$ \\
\hline & & $\mathrm{BC} 2$ & $\mathrm{mg} / \mathrm{kg}$ & 1'550 & $2^{\prime} 780$ & 8 & 19 & 670 & $43.2 \%$ & 82 & 8.2 & 66 & $4.3 \%$ \\
\hline & & $\mathrm{BC} 3$ & $\mathrm{mg} / \mathrm{kg}$ & $32^{\prime} 982$ & $48^{\prime} 000$ & 7 & 18 & $11 ' 354$ & $34.4 \%$ & 1'102 & 10.3 & 862 & $2.6 \%$ \\
\hline Heavy & $\mathrm{Cd}$ & $\mathrm{BC} 1$ & $\mathrm{mg} / \mathrm{kg}$ & 0.06 & - & 5 & 13 & 0.28 & $458.3 \%$ & 0.02 & 18.6 & 0.28 & $458.3 \%$ \\
\hline metals & & $\mathrm{BC} 2$ & $\mathrm{mg} / \mathrm{kg}$ & 0.17 & - & 6 & 15 & 0.13 & $77.7 \%$ & 0.04 & 3.7 & 0.13 & $77.7 \%$ \\
\hline & & $\mathrm{BC} 3$ & $\mathrm{mg} / \mathrm{kg}$ & 3.42 & 3.8 & 9 & 20 & 1.97 & $57.8 \%$ & 0.45 & 4.3 & 0.11 & $3.2 \%$ \\
\hline & $\mathrm{Cr}$ & $\mathrm{BC} 1$ & $\mathrm{mg} / \mathrm{kg}$ & 16.3 & 9.0 & 7 & 16 & 16.4 & $100.7 \%$ & 1.7 & 9.6 & 1.0 & $6.4 \%$ \\
\hline & & $\mathrm{BC} 2$ & $\mathrm{mg} / \mathrm{kg}$ & 8.8 & 7.0 & 7 & 16 & 7.2 & $82.0 \%$ & 1.0 & 7.1 & 0.8 & $8.5 \%$ \\
\hline & & $\mathrm{BC} 3$ & $\mathrm{mg} / \mathrm{kg}$ & 64.2 & 89.0 & 8 & 19 & 19.7 & $30.6 \%$ & 5.5 & 3.6 & 2.5 & $3.9 \%$ \\
\hline & $\mathrm{Cu}$ & $\mathrm{BC} 1$ & $\mathrm{mg} / \mathrm{kg}$ & 12.0 & 11.0 & 10 & 23 & 5.6 & $46.4 \%$ & 1.3 & 4.2 & 1.2 & $10.2 \%$ \\
\hline & & $\mathrm{BC} 2$ & $\mathrm{mg} / \mathrm{kg}$ & 28.7 & 41.0 & 10 & 23 & 11.7 & $41.0 \%$ & 2.8 & 4.2 & 1.2 & $4.2 \%$ \\
\hline & & $\mathrm{BC} 3$ & $\mathrm{mg} / \mathrm{kg}$ & 475.6 & 600.0 & 10 & 23 & 263.2 & $55.4 \%$ & 30.1 & 8.8 & 15.7 & $3.3 \%$ \\
\hline & $\mathrm{Ni}$ & $\mathrm{BC} 1$ & $\mathrm{mg} / \mathrm{kg}$ & 9.4 & 7.0 & 8 & 18 & 8.7 & $91.7 \%$ & 1.1 & 8.0 & 1.1 & $11.3 \%$ \\
\hline & & $\mathrm{BC} 2$ & $\mathrm{mg} / \mathrm{kg}$ & 7.4 & 9.0 & 8 & 18 & 3.3 & $45.0 \%$ & 0.9 & 3.8 & 1.7 & $23.1 \%$ \\
\hline & & $\mathrm{BC} 3$ & $\mathrm{mg} / \mathrm{kg}$ & 60.1 & 66.0 & 9 & 21 & 17.1 & $28.5 \%$ & 5.2 & 3.3 & 2.2 & $3.6 \%$ \\
\hline & $\mathrm{Pb}$ & BC1 & $\mathrm{mg} / \mathrm{kg}$ & 2.0 & 2.0 & 6 & 12 & 1.3 & $65.3 \%$ & 0.3 & 4.5 & 0.7 & $35.9 \%$ \\
\hline & & $\mathrm{BC} 2$ & $\mathrm{mg} / \mathrm{kg}$ & 17.4 & 21.0 & 8 & 18 & 7.7 & $44.4 \%$ & 1.8 & 4.3 & 0.7 & $4.0 \%$ \\
\hline & & $\mathrm{BC} 3$ & $\mathrm{mg} / \mathrm{kg}$ & 135.7 & 170.0 & 8 & 18 & 31.0 & $22.9 \%$ & 10.4 & 3.0 & 1.7 & $1.3 \%$ \\
\hline & $Z n$ & BC1 & $\mathrm{mg} / \mathrm{kg}$ & 42.8 & 59.0 & 9 & 20 & 19.2 & $44.9 \%$ & 3.9 & 4.9 & 2.9 & $6.7 \%$ \\
\hline & & $\mathrm{BC} 2$ & $\mathrm{mg} / \mathrm{kg}$ & 57.4 & 86.0 & 10 & 23 & 38.5 & $67.1 \%$ & 5.0 & 7.7 & 4.9 & $8.5 \%$ \\
\hline & & $\mathrm{BC} 3$ & $\mathrm{mg} / \mathrm{kg}$ & $2^{\prime} 047.8$ & $3^{\prime} 000.0$ & 10 & 23 & 805.8 & $39.4 \%$ & 104.0 & 7.7 & 39.3 & $1.9 \%$ \\
\hline PAH & $P A H$ & $\mathrm{BC} 1$ & $\mathrm{ng} / \mathrm{g}$ & $2^{\prime} 893$ & $6^{\prime} 004$ & 5 & 12 & 1'951 & $67.4 \%$ & 394 & 4.9 & 175 & $6.1 \%$ \\
\hline & SUM & $\mathrm{BC} 2$ & $\mathrm{ng} / \mathrm{g}$ & $2^{\prime} 252$ & 3'553 & 5 & 13 & $1 ' 297$ & $57.6 \%$ & 319 & 4.1 & 144 & $6.4 \%$ \\
\hline & & $\mathrm{BC} 3$ & $\mathrm{ng} / \mathrm{g}$ & 843 & 962 & 5 & 13 & 336 & $39.9 \%$ & 138 & 2.4 & 49 & $5.8 \%$ \\
\hline & $P A H$ & $\mathrm{BC} 1$ & $\mathrm{ng} / \mathrm{g}$ & 21 & 184 & 5 & 12 & 30 & $139.7 \%$ & 6 & 4.9 & 1 & $6.9 \%$ \\
\hline & $A C E$ & $\mathrm{BC} 2$ & $\mathrm{ng} / \mathrm{g}$ & 24 & 41 & 5 & 13 & 34 & $145.3 \%$ & 7 & 5.2 & 1 & $4.7 \%$ \\
\hline & & $\mathrm{BC} 3$ & $\mathrm{ng} / \mathrm{g}$ & 8 & 10 & 5 & 12 & 6 & $73.4 \%$ & 3 & 2.2 & 1 & $12.4 \%$ \\
\hline & $P A H$ & $\mathrm{BC} 1$ & $\mathrm{ng} / \mathrm{g}$ & 82 & 82 & 5 & 12 & 42 & $51.6 \%$ & 19 & 2.2 & 6 & $7.0 \%$ \\
\hline & $A C Y$ & $\mathrm{BC} 2$ & $\mathrm{ng} / \mathrm{g}$ & 51 & 10 & 5 & 13 & 38 & $75.5 \%$ & 13 & 3.0 & 1 & $2.1 \%$ \\
\hline & & $\mathrm{BC} 3$ & $\mathrm{ng} / \mathrm{g}$ & 10 & 10 & 5 & 13 & 9 & $99.0 \%$ & 3 & 3.1 & 4 & $39.8 \%$ \\
\hline & $P A H$ & $\mathrm{BC} 1$ & $\mathrm{ng} / \mathrm{g}$ & 91 & 194 & 4 & 11 & 95 & $104.6 \%$ & 21 & 4.6 & 5 & $6.0 \%$ \\
\hline & $A N T$ & $\mathrm{BC} 2$ & $\mathrm{ng} / \mathrm{g}$ & 65 & 184 & 5 & 13 & 69 & $106.4 \%$ & 16 & 4.4 & 6 & $9.8 \%$ \\
\hline & & $\mathrm{BC} 3$ & $\mathrm{ng} / \mathrm{g}$ & 29 & 41 & 5 & 13 & 21 & $71.6 \%$ & 8 & 2.6 & 2 & $6.5 \%$ \\
\hline
\end{tabular}


Table 1. continued

\begin{tabular}{|c|c|c|c|c|c|c|c|c|c|c|c|c|c|}
\hline \multirow[t]{2}{*}{ Group } & \multirow{2}{*}{$\begin{array}{l}\text { Para- } \\
\text { meter }\end{array}$} & \multirow{2}{*}{ Sample } & \multirow[t]{2}{*}{ Unit } & \multirow[t]{2}{*}{ Mean value } & \multirow{2}{*}{$\begin{array}{l}\text { Reference } \\
\text { value }\end{array}$} & \multicolumn{2}{|c|}{ Number of } & \multicolumn{2}{|c|}{ Reproducibility SD } & \multicolumn{2}{|c|}{ Horwitz function } & \multicolumn{2}{|c|}{ Repeatability SD } \\
\hline & & & & & & labs & results & absolute & relative & value & ratio & absolute & relative \\
\hline & $P A H$ & $\overline{\mathrm{BC} 1}$ & $\mathrm{ng} / \mathrm{g}$ & 64 & 133 & 5 & 12 & 53 & $83.2 \%$ & 15 & 3.4 & 4 & $6.3 \%$ \\
\hline & \multirow[t]{2}{*}{$B A A$} & $\mathrm{BC} 2$ & $\mathrm{ng} / \mathrm{g}$ & 71 & 164 & 5 & 13 & 66 & $92.2 \%$ & 17 & 3.9 & 6 & $7.8 \%$ \\
\hline & & $\mathrm{BC} 3$ & $\mathrm{ng} / \mathrm{g}$ & 33 & 41 & 5 & 13 & 29 & $88.0 \%$ & 9 & 3.3 & 3 & $8.9 \%$ \\
\hline & \multirow{3}{*}{$\begin{array}{l}P A H \\
B A P\end{array}$} & $\mathrm{BC} 1$ & $\mathrm{ng} / \mathrm{g}$ & 60 & 82 & 5 & 12 & 34 & $56.9 \%$ & 15 & 2.3 & 2 & $3.0 \%$ \\
\hline & & $\mathrm{BC} 2$ & $\mathrm{ng} / \mathrm{g}$ & 58 & 113 & 5 & 13 & 51 & $88.1 \%$ & 14 & 3.6 & 6 & $9.9 \%$ \\
\hline & & $\mathrm{BC} 3$ & $\mathrm{ng} / \mathrm{g}$ & 28 & 31 & 5 & 13 & 24 & $85.4 \%$ & 8 & 3.1 & 3 & $9.4 \%$ \\
\hline & \multirow{3}{*}{$\begin{array}{l}P A H \\
B B F\end{array}$} & $\mathrm{BC} 1$ & $\mathrm{ng} / \mathrm{g}$ & 61 & 82 & 5 & 12 & 43 & $71.0 \%$ & 15 & 2.9 & 8 & $13.7 \%$ \\
\hline & & $\mathrm{BC} 2$ & $\mathrm{ng} / \mathrm{g}$ & 51 & 103 & 5 & 13 & 25 & $49.4 \%$ & 13 & $\underline{2.0}$ & 6 & $11.7 \%$ \\
\hline & & $\mathrm{BC} 3$ & $\mathrm{ng} / \mathrm{g}$ & 15 & 21 & 5 & 13 & 11 & $69.7 \%$ & 5 & 2.3 & 2 & $13.3 \%$ \\
\hline & \multirow{3}{*}{$\begin{array}{l}P A H \\
B K F\end{array}$} & BC1 & $\mathrm{ng} / \mathrm{g}$ & 28 & 51 & 5 & 12 & 24 & $84.6 \%$ & 8 & 3.1 & 1 & $4.6 \%$ \\
\hline & & $\mathrm{BC} 2$ & $\mathrm{ng} / \mathrm{g}$ & 13 & 62 & 5 & 13 & 5 & $35.7 \%$ & 4 & $\underline{1.2}$ & 1 & $6.9 \%$ \\
\hline & & $\mathrm{BC} 3$ & $\mathrm{ng} / \mathrm{g}$ & 7 & 10 & 5 & 13 & 3 & $45.0 \%$ & 2 & $\underline{1.3}$ & 1 & $7.4 \%$ \\
\hline & \multirow{3}{*}{$\begin{array}{l}P A H \\
B P E\end{array}$} & $\mathrm{BC} 1$ & $\mathrm{ng} / \mathrm{g}$ & 34 & 31 & 3 & 8 & 41 & $120.0 \%$ & 9 & 4.5 & 6 & $16.6 \%$ \\
\hline & & $\mathrm{BC} 2$ & $\mathrm{ng} / \mathrm{g}$ & 24 & 51 & 5 & 13 & 18 & $76.1 \%$ & 7 & 2.7 & 2 & $10.6 \%$ \\
\hline & & $\mathrm{BC} 3$ & $\mathrm{ng} / \mathrm{g}$ & 15 & 21 & 5 & 13 & 6 & $36.3 \%$ & 5 & $\underline{1.2}$ & 2 & $10.6 \%$ \\
\hline & \multirow{3}{*}{$\begin{array}{l}\text { PAH } \\
\text { CHR }\end{array}$} & $\mathrm{BC} 1$ & $\mathrm{ng} / \mathrm{g}$ & 64 & 163 & 5 & 12 & 70 & $109.5 \%$ & 16 & 4.5 & 6 & $9.3 \%$ \\
\hline & & $\mathrm{BC} 2$ & $\mathrm{ng} / \mathrm{g}$ & 84 & 215 & 5 & 13 & 95 & $112.9 \%$ & 20 & 4.9 & 8 & $10.0 \%$ \\
\hline & & $\mathrm{BC} 3$ & $\mathrm{ng} / \mathrm{g}$ & 39 & 82 & 5 & 13 & 49 & $123.4 \%$ & 10 & 4.7 & 4 & $10.4 \%$ \\
\hline & \multirow{3}{*}{$\begin{array}{l}P A H \\
D B A\end{array}$} & $\mathrm{BC} 1$ & $\mathrm{ng} / \mathrm{g}$ & 6 & 7 & 3 & 8 & 7 & $119.5 \%$ & 2 & 3.4 & 1 & $24.0 \%$ \\
\hline & & $\mathrm{BC} 2$ & $\mathrm{ng} / \mathrm{g}$ & 6 & 10 & 4 & 9 & 11 & $191.0 \%$ & 2 & 5.5 & 2 & $35.8 \%$ \\
\hline & & $\mathrm{BC} 3$ & $\mathrm{ng} / \mathrm{g}$ & 5 & 6 & 4 & 9 & 4 & $94.5 \%$ & 2 & 2.6 & 2 & $35.7 \%$ \\
\hline & $P A H$ & $\mathrm{BC} 1$ & $\mathrm{ng} / \mathrm{g}$ & 132 & 356 & 5 & 12 & 158 & $119.3 \%$ & 29 & 5.5 & 12 & $9.0 \%$ \\
\hline & $F L A$ & $\mathrm{BC} 2$ & $\mathrm{ng} / \mathrm{g}$ & 109 & 328 & 6 & 14 & 88 & $80.9 \%$ & 24 & 3.6 & 10 & $8.9 \%$ \\
\hline & & $\mathrm{BC} 3$ & $\mathrm{ng} / \mathrm{g}$ & 48 & 82 & 5 & 13 & 28 & $59.7 \%$ & 12 & 2.4 & 3 & $6.1 \%$ \\
\hline & $P A H$ & $\mathrm{BC} 1$ & $\mathrm{ng} / \mathrm{g}$ & 33 & 82 & 5 & 12 & 31 & $91.9 \%$ & 9 & 3.4 & 1 & $3.4 \%$ \\
\hline & $F L U$ & $\mathrm{BC} 2$ & $\mathrm{ng} / \mathrm{g}$ & 28 & 62 & 5 & 13 & 22 & $78.7 \%$ & 8 & 2.9 & 1 & $4.5 \%$ \\
\hline & & $\mathrm{BC} 3$ & $\mathrm{ng} / \mathrm{g}$ & 11 & 10 & 5 & 13 & 2 & $\underline{18.4 \%}$ & 3 & $\underline{0.6}$ & 2 & $15.1 \%$ \\
\hline & $P A H$ & $\mathrm{BC} 1$ & $\mathrm{ng} / \mathrm{g}$ & 34 & 20 & 3 & 8 & 17 & $49.7 \%$ & 9 & $\underline{1.9}$ & 3 & $8.6 \%$ \\
\hline & $I N D$ & $\mathrm{BC} 2$ & $\mathrm{ng} / \mathrm{g}$ & 33 & 51 & 5 & 13 & 40 & $120.4 \%$ & 9 & $\overline{4.5}$ & 4 & $11.4 \%$ \\
\hline & & $\mathrm{BC} 3$ & $\mathrm{ng} / \mathrm{g}$ & 14 & 10 & 5 & 13 & 10 & $75.0 \%$ & 4 & 2.5 & 2 & $16.3 \%$ \\
\hline & $P A H$ & $\mathrm{BC} 1$ & $\mathrm{ng} / \mathrm{g}$ & $1 ' 341$ & 3'364 & 6 & 13 & 954 & $71.2 \%$ & 205 & 4.7 & 94 & $7.0 \%$ \\
\hline & $N A P$ & $\mathrm{BC} 2$ & $\mathrm{ng} / \mathrm{g}$ & 896 & $1^{\prime} 025$ & 6 & 14 & 754 & $84.1 \%$ & 146 & 5.2 & 36 & $4.1 \%$ \\
\hline & & BC3 & $\mathrm{ng} / \mathrm{g}$ & 376 & 412 & 6 & 14 & 94 & $25.0 \%$ & 70 & $\underline{1.3}$ & 33 & $8.8 \%$ \\
\hline & $P A H$ & $\mathrm{BCl}$ & $\mathrm{ng} / \mathrm{g}$ & 405 & 816 & 5 & 12 & 515 & $127.0 \%$ & 74 & 6.9 & 72 & $17.8 \%$ \\
\hline & PHE & $\mathrm{BC} 2$ & $\mathrm{ng} / \mathrm{g}$ & 341 & 816 & 6 & 14 & 323 & $94.7 \%$ & 64 & 5.0 & 38 & $11.1 \%$ \\
\hline & & $\mathrm{BC} 3$ & $\mathrm{ng} / \mathrm{g}$ & 94 & 82 & 6 & 14 & 56 & $60.0 \%$ & 21 & 2.6 & 18 & $18.8 \%$ \\
\hline & $P A H$ & BC1 & $\mathrm{ng} / \mathrm{g}$ & 149 & 357 & 5 & 12 & 216 & $145.2 \%$ & 32 & 6.8 & 8 & $5.1 \%$ \\
\hline & $P Y R$ & $\mathrm{BC} 2$ & $\mathrm{ng} / \mathrm{g}$ & 136 & 318 & 5 & 13 & 114 & $83.3 \%$ & 29 & 3.9 & 10 & $7.7 \%$ \\
\hline & & $\mathrm{BC} 3$ & $\mathrm{ng} / \mathrm{g}$ & 61 & 93 & 5 & 13 & 42 & $68.8 \%$ & 15 & 2.8 & 6 & $9.2 \%$ \\
\hline Further & $\underline{\mathrm{pH}}$ & $\mathrm{BC} 1$ & & 9.91 & 8.3 & 19 & 44 & 0.56 & $\underline{5.7 \%}$ & & - & 0.03 & $0.3 \%$ \\
\hline para- & & $\mathrm{BC} 2$ & & 9.30 & 8.3 & 19 & 44 & 0.42 & $4.5 \%$ & & - & 0.03 & $0.3 \%$ \\
\hline meters & & $\mathrm{BC} 3$ & & 7.09 & 7.1 & 19 & 44 & 0.47 & $6.6 \%$ & & - & 0.06 & $0.9 \%$ \\
\hline & $E C$ & $\mathrm{BC} 1$ & $\mathrm{uS} / \mathrm{cm}$ & $1 ' 203$ & 989 & 13 & 33 & 826 & $68.7 \%$ & & - & 31 & $2.6 \%$ \\
\hline & & $\mathrm{BC} 2$ & $\mathrm{uS} / \mathrm{cm}$ & 1'054 & 759 & 13 & 33 & 736 & $69.8 \%$ & & - & 16 & $1.5 \%$ \\
\hline & & $\mathrm{BC} 3$ & $\mathrm{uS} / \mathrm{cm}$ & 785 & 617 & 13 & 33 & 530 & $67.5 \%$ & & - & 16 & $2.0 \%$ \\
\hline & SSA & $\mathrm{BC} 1$ & $\mathrm{~m} 2 / \mathrm{g}$ & 316.0 & 224.8 & 8 & 13 & 58.3 & $\underline{18.5 \%}$ & & - & 0.4 & $0.1 \%$ \\
\hline & & $\mathrm{BC} 2$ & $\mathrm{~m} 2 / \mathrm{g}$ & 97.8 & 63.8 & 8 & 13 & 28.7 & $29.4 \%$ & & - & 1.6 & $1.7 \%$ \\
\hline & & $\mathrm{BC} 3$ & $\mathrm{~m} 2 / \mathrm{g}$ & 56.5 & 25.6 & 8 & 13 & 11.5 & $20.4 \%$ & & - & 0.2 & $0.4 \%$ \\
\hline
\end{tabular}

${ }^{\mathrm{a}}$ The fundamental statistical evaluation was made according to DIN 38402 A45 with Hampel-estimator for the mean and Q-estimator for the standard deviation (SD). The results of LAB_04 were taken as reference values obtained with the methods currently recommended by the EBC for biochar analysis. The values of the Horwitz function were calculated for the mean absolute and relative to the mean. The Horwitz ratio (HorRat) is the quotient of the absolute reproducibility SD divided by the Horwitz value. As the Horwitz function is defined for concentrations, there are no values for $\mathrm{pH}, \mathrm{EC}$, and SSA. The repeatability is calculated as the mean of standard deviations of multiple results from laboratories for the same parameter (mean variation within laboratories). The font style of the analytical parameters is defined in dependence of their mean relative reproducibility SD: $\leqq \mathbf{1 0} \%$, $\mathbf{1 0 - 2 0 \%}, 20-40 \%,>40 \%$. The font style of the single values of the relative reproducibility SD is defined as $\leq \mathbf{1 0} \%, 10-20 \%, 20-40 \%,>40 \%$, of the Horwitz ratio as $\leq \mathbf{2}, \mathbf{2 - 4}, \mathbf{4 - 8},>8$ and of the repeatability SD as $<10 \%$, $10-20 \%,>20 \%$. All mass concentrations are given on a dry weight (dw) basis.

However, it is difficult to conceive that residual water accounted for all of the observed divergence. With the exception of two measurements, the values of BC3 were normally distributed (Figure S2, p 205).

Relative reproducibility SD between 4 and $6 \%$ and good laboratory precisions (relative repeatability SD) between 0.6 and $2.8 \%$ were achieved. This is most likely because elemental analysis via combustion was performed by means of commercially available instrumentation with highly standardized methods. The HorRat was low (between 1.5 and 3.1) compared to most other parameters of this interlaboratory comparison (Table 1). The high $\mathrm{C}$ content of the samples compared to any other element certainly facilitated precision. Correspondingly, mean values obtained in the interlaboratory comparison were very close to the reference values. However, in particular if microanalysis is used, sample inhomogeneity (despite the advanced sampling method), slight deviations during weighing of the samples and the reference material, 
as well as hygroscopic properties of the chars can increase deviations of the results.

Hydrogen (H). During pyrolysis, dehydration leads to condensation and thus increasing aromatization of the sample. ${ }^{17}$ Accordingly, the atomic $\mathrm{H} / \mathrm{C}_{\text {org }}$ ratio is used as an index for the aromaticity ${ }^{18}$ and the carbonization degree of the biochar. ${ }^{1,10}$ Because it is further assumed that stability of biochars increases with aromatization, ${ }^{19,20}$ this ratio represents an important parameter to predict their biochemical recalcitrance. However, applying this parameter, one has to bear in mind that $\mathrm{H}$ in minerals and/or adsorbed in the form of capillary water can give misleading results.

Similar to the $\mathrm{C}$ contents, the $\mathrm{H}$ concentrations were measured by elemental analysis (Table S1). In general, the mean values obtained within the interlaboratory comparison were only slightly higher than the reference measurements (Table 1 ). The $\mathrm{Zu}$ scores of all three samples were $<|3|$ for all laboratories (Figure 1), confirming that no major deviations in the reported data occurred. Correspondingly, all box plots (Figure S1, pp 12, $52,92)$ were within the limits of tolerance. The Kernel density distributions (Figure S2, pp 132, 172, 212) were normal with a right-side shoulder for $\mathrm{BC} 1$ and a bimodal distribution for $\mathrm{BC} 2$. A broad but still unimodal distribution was observed for BC3. Relative reproducibility SD (15-21\%) and relative repeatability SD $(2.7-8.2 \%)$ were $>3$ times higher than for $C$, whereas the HorRat values were approximately twice as high (Table 1 ).

Nitrogen $(N)$. The $\mathrm{N}$ content of charred residues greatly depends upon the feedstock and increases with the use of organic N-rich sources. Up to pyrolysis temperatures of $600{ }^{\circ} \mathrm{C}$, no major heat-induced alteration of the $\mathrm{C} / \mathrm{N}$ ratio was observed. $^{21}$ It was shown that pyrolysis turns the peptideous residues into so-called "black nitrogen", which is mainly composed of $\mathrm{N}$-heteroaromatic structures. ${ }^{22}$

The $\mathrm{N}$ contents used in the present statistical evaluation derived mainly from methods using elemental analysis by dry combustion (Table S1). Only one laboratory (LAB_06) applied the Kjeldahl method. BC1 resulted in $\mathrm{N}$ contents $($ mean $=0.35 \%)$ that are typical for wood biochars; higher values were reported for BC2 (1.4\%) and BC3 (2.1\%). The latter yielded an atomic $\mathrm{C} / \mathrm{N}$ ratio of 10 , typical for sewage sludge chars. The mean and reference values were comparable (Table 1). Whereas the data based on elemental analysis were in the $\mathrm{Zu}$ score $<|3|$ tolerance limit (Figure 1), a clear discrepancy was evident for the data obtained with the Kjeldahl method, which consistently delivered the lowest $\mathrm{N}$ concentrations (Figure S1, pp 16, 56, 96). Correspondingly, a bimodal Kernel distribution was observed for BC2 and BC3 (Figure S2, pp 136, $176,216)$. Except for $\mathrm{BC} 1(59 \%)$, good relative reproducibility $\mathrm{SD}$ values of 14 and $8 \%$ for $\mathrm{BC} 2$ and $\mathrm{BC} 3$, respectively, were obtained. Relative repeatability $\mathrm{SD}$ values were satisfying and ranged from 1.9 to $5.6 \%$. Correspondingly, the HorRat of $\mathrm{BC} 1$ was highest (12.4) and more acceptable for BC2 (3.8) and BC3 (2.1).

Oxygen (O). The $\mathrm{O}$ contents were either measured by direct elemental analysis or indirectly calculated (Table S1). For the latter, the conventional DIN and ASTM methods suggest estimating organic oxygen concentration by subtracting the contributions of determined $\mathrm{C}, \mathrm{H}, \mathrm{S}$, and high-temperature ash from the total dw. However, subjecting the data of the interlaboratory comparison to statistical analysis, one has to bear in mind that only four laboratories contributed to its determination. As a first consequence of the two methodological approaches in use, the mean and reference values differed by up to a factor of 2.8 (Table 1 ). Although the $\mathrm{Zu}$ scores were $<|3|$ for all participating laboratories (Figure 1) and normal Kernel distributions were obtained for all three samples (Figure S2, pp $139,179,219)$, the relative reproducibility SD and the relative repeatability SD for this parameter varied within 35-119 and 2.9-9.9\%, respectively, considerably more than for $\mathrm{C}$ or $\mathrm{H}$ contents (Table 1 ). This high variability was also reflected by elevated HorRat values between 11.5 and 42.4. Due to the low amount of data points, it cannot be decided if calculation or direct measurement represents a more appropriate standard method for the characterization of biochar.

Sulfur (S). The determination of $S$ does not represent a standard feature of commonly available elemental analyzers. Correspondingly, only five laboratories contributed their values (Table S1). Zu scores indicated analytical difficulties for all samples in at least some laboratories (Figure 1). The $S$ content was lowest in BC1 (mean value $=0.04 \%$ ) and probably close to the detection limits of the instruments. This may have been the reason for reproducibility problems, indicated by a relative reproducibility SD as high as $161 \%$, a HorRat of 22.3 , and a multimodal Kernel distribution (Figure S2, p 160). Corresponding determinations with ICP-OES improved the Kernel distribution to a bimodal plot (Figure S2, p 161). BC2 revealed a slightly higher $\mathrm{S}$ content with comparable mean values obtained with both methods (elemental analysis, $0.12 \%$; ICPOES, $0.13 \%$; Table 1), but only the data recorded by ICP-OES resulted in a normal Kernel distribution (Figure S2, pp 200, 201). Reproducibility numbers were mediocre in both cases (e.g., relative reproducibility SD of 39 and $85 \%$ and HorRat of 7.5 and 15.6 for elemental analysis and ICP-OES, respectively; Table 1).

Proteins in sewage sludge provide an additional source of organic $S$ in the respective charred residues. However, only little is known about its speciation ${ }^{23}$ and how pyrolytic $S$ affects plant growth. ${ }^{24}$ Compared to $\mathrm{BC} 1$ and $\mathrm{BC} 2$, higher mean $\mathrm{S}$ concentrations of 0.7 and $0.6 \%$ were determined for $\mathrm{BC} 3$ via elemental analysis and ICP-OES, respectively (Table 1). Both numbers were slightly below the reference value. Kernel density plots showed tri- and bimodal distributions (Figure S2, pp 240, 241). Reproducibility parameters of BC3 were better than for BC1 and BC2 (e.g., relative reproducibility SD of 25 and $29 \%$ and HorRat of 5.9 and 6.8 for elemental analysis and ICP-OES, respectively; Table 1). Thus, it can be concluded that both techniques face sensitivity problems if biochars from feedstocks with low $S$ contents are analyzed. At $S$ concentrations $>0.1 \%$, relatively reliable and comparable values were obtained with both methods.

Ash. Degradation of organic components during pyrolysis results in a relative enrichment of ash. Although ash can be a source of metals or silicates when applied to soil, it is also a source of important plant nutrients. The biochars used here showed mean ash contents varying from 11 to $72 \%$ (Table 1 ). The highest number was obtained for $\mathrm{BC} 3$ derived from sewage sludge. Besides the fact that household sewage is rich in minerals, runoff from streets and gardens containing soil material, mineral-precipitating agents, and sand probably contributed to its high ash content.

The $\mathrm{Zu}$ scores were $<|3|$ for all laboratories and for all three biochars, revealing that no major deviations of the reported data occurred (Figure 1). The reference values were slightly higher than the mean (Table 1). The box plots (Figure S1, pp $4,44,84)$ indicate that the ash content generally decreased with increasing combustion temperature. Either lower temperatures 
did not lead to a complete combustion of all organic residues or, which is more likely, thermal destruction of the remaining mineral phase occurred at higher temperatures, because most carbonates are destroyed between 600 and $900{ }^{\circ} \mathrm{C}$ and their $\mathrm{C}$ is lost as $\mathrm{CO}_{2}$. Note that it is a standard practice in soil and fossil coal analyses to use combustion at $550{ }^{\circ} \mathrm{C}$ for total ash content and to introduce then a second carbon analysis at $950{ }^{\circ} \mathrm{C}$ for the determination of the carbonate content. ${ }^{25}$

As can be expected, relative reproducibility SD, HorRat, and relative repeatability SD values improved with increasing ash content and ranged from 32 to $2 \%$, from 11.7 to 1.0 , and from 9.5 to $0.3 \%$, respectively (Table 1 ). Such values can be considered satisfactory.

Summary of $C, H, N, O, S$, and Ash Analysis. The use of automated combustion analysis to determine CHNOS in biochar is clearly the method of choice that led to highly reproducible results for the important parameters of $\mathrm{C}$ and $\mathrm{H}$ contents. The determinations of $\mathrm{N}, \mathrm{O}$, and $\mathrm{S}$ concentrations were less precise, especially at low concentrations. Determination of $\mathrm{N}$ by the Kjeldahl method turned out to have less reproducibility when compared to the other methods. However, this observation cannot be generalized because only one laboratory provided the respective data.

Ash contents depended upon the temperature during muffling. Our data indicate that temperatures $>550{ }^{\circ} \mathrm{C}$ resulted in lower ash yields, possibly due to thermal degradation of the mineral phase. On the basis of this observation, the application of the standardized temperature of $550{ }^{\circ} \mathrm{C}$ is recommended for obtaining ash contents of biochars. To determine the content of carbonate and thus of $\mathrm{C}_{\text {org }}$, a second ashing may be performed at temperatures $>950{ }^{\circ} \mathrm{C}$ or via $\mathrm{HCl}$ extraction. Mean values of $\mathrm{C}, \mathrm{N}, \mathrm{H}, \mathrm{S}$, and ash concentrations calculated from the interlaboratory comparison were comparable to the respective reference values, allowing the conclusion that the reference methods ${ }^{8}$ yield representative data for biochars.

Main Elements ( $\mathrm{P}, \mathrm{K}, \mathrm{Na}, \mathrm{Mg}, \mathrm{Mn}, \mathrm{Ca}, \mathrm{Fe})$. General Remarks. Most of the main elements, $\mathrm{P}, \mathrm{K}, \mathrm{Na}, \mathrm{Mg}, \mathrm{Mn}, \mathrm{Ca}$, and $\mathrm{Fe}$, play important roles as plant nutrients in agriculture, although in biochar their contents are rather low and mostly not considered as being of agronomic relevance. ${ }^{2}$ However, even if plant nutrition is not the primary purpose of biochar application, those elements have to be considered for regulatory requirements. For example, biochar is admitted by Austrian, Italian, and Swiss authorities as a soil improver. This implies that the total concentrations of $\mathrm{P}, \mathrm{K}, \mathrm{Mg}$, and $\mathrm{Ca}$ have to be analyzed for control purposes, although they cannot be considered as directly bioavailable. The same holds for $\mathrm{Na}$, $\mathrm{Fe}$, and $\mathrm{Mn}$, which were analyzed due to their relevance in agriculture.

The $\mathrm{Zu}$ scores of these elements do not attract special attention, as almost all of them were clearly $<|3|$ (Table 1) and showed decreasing values for higher concentrations, as expected. For simplicity, elements that show similar patterns are grouped together in the same subsection.

Phosphorus $(P)$. Depending on the feedstock, total $\mathrm{P}$ concentrations were 765 and $6610 \mathrm{mg} / \mathrm{kg}$ for $\mathrm{BC} 1$ and $\mathrm{BC} 2$, respectively, and $60531 \mathrm{mg} / \mathrm{kg}$ for the pyrolyzed sewage sludge (BC3) (Table 1), which extends into the domain of fertilizer contents. The relative reproducibility SD of the results (16-20\%) and the HorRat (3.4-5.4) (Table 1) indicate an expanded distribution of the laboratory values, which is confirmed by the box plots (Figure S1, pp 20,60, 100) and the Kernel density plots (Figure S1, pp 140, 180, 220). As the sequence of the laboratory results in ascending order in the box plots is similar for all samples and the relative repeatability SD was only $\leq 1.2 \%$, it is obvious that there were some systematic differences between the results of the laboratories. Varying methods used for sample preparation and digestion (Table S1) could explain the discrepancies, although a fully consistent attribution of the results is not possible. It can be expected that the reference method for digestion recommended by the $\mathrm{EBC}^{8}$ (fusion with $\mathrm{LiBO}_{2}$ at $1050{ }^{\circ} \mathrm{C}$ and dissolution in $\mathrm{HCl}$ ) led to a complete destruction of the matrix of biochar so that all of the phosphorus in the sample was analyzed. This is visible in the box plots (Figure S1, pp 20, 60, 100), where the reference value is systematically higher than the mean. Next in descending order of digestion power would be aqua regia extraction in a microwave system. The results of LAB_08 confirm this hypothesis, whereas those of LAB_13 were below the mean. This may be explained by diverting digestion temperatures and programs. The remaining two digestion methods were less powerful, as visualized by the box plots of $\mathrm{BC} 2$ and $\mathrm{BC} 3$ (Figure S1, pp 60, 100), although the Kernel density plots do not confirm the significance of this difference (Figure S2, pp 180, 220).

Potassium ( $\mathrm{K}$ ) and Sodium (Na). The total concentrations of $\mathrm{K}$ and $\mathrm{Na}$ in the three samples were in a narrow range (K, 4096-10016 mg/kg; Na, 103-1273 mg/kg) (Table 1). The pattern of the data was very similar to that of $\mathrm{P}$, but much more pronounced. The relative reproducibility SD of the results (K, 59-69\%; Na, 10-51\%) and the HorRat (K, 12.9-17.1; Na, 1.8-6.3) were far from being "fit for purpose", whereas the within-laboratory repeatability $\mathrm{SD}$ values were good $(\mathrm{K}$, 1.3-1.8\%; $\mathrm{Na}, 2.1-7.8 \%$ ). For $\mathrm{K}$, the Kernel density plots (Figure S2, pp 133, 173, 213) show an extended distribution except for $\mathrm{BC} 3$, where the reference value has its own mode and for $\mathrm{Na}$, a trimodal distribution. The systematic differences between laboratories are also clearly visible in the box plots $(\mathrm{K}$, Figure S1, pp 13, 53, 93; Na, Figure S1, pp 17, 57, 97) and can at least partly be explained by the respective analytical methods: the fusion method, used by LAB_04 only, is the only one that completely releases $\mathrm{K}$ and $\mathrm{Na}$ (and other elements) from the matrix $^{26,27}$ and delivered much higher values for all three biochars (Figure S1, pp 13, 17, 53, 57, 93, 97; ref value). However, most participants of the interlaboratory comparison applied acid digestion with aqua regia or nitric acid with microwave heating, which keep both alkaline metals to a certain extent enclosed in the matrix. ${ }^{27}$ For $\mathrm{BC} 3$, originating from sewage sludge with high silica and ash content, the fusion method delivered much higher values than the acid extraction. Adding hydrogen peroxide to acid did not lead to a higher degree of digestion for $\mathrm{K}$ and $\mathrm{Na}$, as evidenced by the results of $L A B \quad 05$ and $L A B \quad 21$. Calcination and acid extraction of the ash can also be useful for the digestion, but only if the samples contain high amounts of $\mathrm{C}_{\text {org }}$ and have low contributions of ash (results of $\mathrm{LAB} 11$ for $\mathrm{BC1}$ ).

Magnesium $(\mathrm{Mg})$ and Manganese $(\mathrm{Mn})$. The variability of $\mathrm{Mg}$ and $\mathrm{Mn}$ concentrations within the three samples was quite small ( $\mathrm{Mg}, 1724-8877 \mathrm{mg} / \mathrm{kg} ; \mathrm{Mn}, 127-514 \mathrm{mg} / \mathrm{kg})$. The relative reproducibility SD values of $\mathrm{BC} 1$ and $\mathrm{BC} 2(\mathrm{Mg}, 28$ and $19 \% ; \mathrm{Mn}, 22$ and $32 \%$ ) were comparable to the one of $\mathrm{P}$, whereas the one for BC3 ( $\mathrm{Mg}, 51 \%$; Mn, 44\%) was similar to that for $\mathrm{K}$ (Table 1). The same holds for the HorRat ( $\mathrm{Mg}, 5.4$, 4.0, and 12.6; $\mathrm{Mn}, 3.2,4.2$, and 7.0). The relative repeatability SD (Mg, 1.5-3.8\%; Mn, 1.5-3.7\%) was much smaller than the relative reproducibility $\mathrm{SD}$, which points to the influence of the 
different digestion methods. This is also visible in the Kernel density plots, where BC1 and BC2 (Figure S2, pp 134, 135, $174,175)$ showed at least two modes of results. This type of influence was obviously systematic, as the sequence of the laboratory results in ascending order in the box plots was almost the same for all three samples (Figure S1, pp 14, 15, 54, $55,94,95)$, although to a different extent, depending on the feedstock (BC3 > BC1, BC2).

Calcium (Ca). The Ca concentrations covered a range from 21705 to $62219 \mathrm{mg} / \mathrm{kg}$ and showed a pattern comparable to that of K. Relative reproducibility SD values (45-65\%) as well as the HorRat (14.7-18.2) were too high to be "fit for purpose", whereas the relative repeatability SD (1.0-4.5\%) was in the expected range (Table 1$)$. The similar ascending order of box plots for Ca (Figure S1, pp 6, 46, 86) and Mg (Figure S1, pp 14, 54, 94) indicates the same type of influence by the digestion methods, which is explained by the similarity of their properties, both being alkaline earth metals. The Kernel density plot of BC3 (Figure S2, p 206) with its two distinctive modes, emphasizes the methodological differences. This was less pronounced for $\mathrm{BC} 1$ and $\mathrm{BC} 2$, which exhibited unimodal Kernel plots (Figure S2, pp 126, 166).

Iron (Fe). The $\mathrm{Fe}$ concentrations ranged between 1087 and $32982 \mathrm{mg} / \mathrm{kg}$ and were dependent on the biochar feedstock (Table 1). The relative reproducibility SD (34-45\%) and the HorRat $(8.1-10.3)$ were quite elevated. The Kernel density plots of $\mathrm{BC} 1$ and $\mathrm{BC} 3$ were clearly bimodal, whereas $\mathrm{BC} 2$ resulted in a broadened normal distribution (Figure S2, pp 131, 211 , and 171 , respectively). Together with the relatively small relative repeatability SD (2.6-4.3\%), this suggests a methodological impact of the digestion on the results. Also, the sequence of the laboratory results in ascending order was not as consistent as for the other elements (Figure S1, pp 11, 51, 91). Due to the small database, a quantification of the methodological influence is not possible. Obviously, the fusion method used by LAB_04 led to results that were systematically higher than those of the others. Only this method is capable of releasing Fe from the matrix completely, similar to the alkaline metals. $^{27}$ Acidic extraction with microwave heating was less efficient, and the degree of digestion depended on the type of biochar. High ash contents increased the fraction of recalcitrant $\mathrm{Fe}$, especially in $\mathrm{BC} 3$, where $\mathrm{Fe}$ accumulated in its feedstock, sewage sludge, as a result of its use for phosphate precipitation in wastewater treatment. Although the analysis of $\mathrm{Fe}$ is not required by any regulation, its content influences, for example, the redox behavior, paramagnetism, and contaminant sorption mechanisms of biochar. ${ }^{28,29}$ In cases when such properties are explored, more exhaustive methods such as the fusion method may be required.

Summary of Main Elements $(P, K, \mathrm{Na}, \mathrm{Mg}, \mathrm{Mn}, \mathrm{Ca}, \mathrm{Fe})$. The observed differences in the concentrations of the main elements can mainly be attributed to the use of differing digestion methods. The respective reference method suggested by the $\mathrm{EBC}$ (fusion with $\mathrm{LiBO}_{2}$ and dissolution in $\left.\mathrm{HCl}\right)^{8}$ consistently led to the most efficient extraction; however, this method is more technical and laborious than other digestion methods. From a practical point of view, the aqua regia extraction in a microwave system is a worthy alternative that offers the possibility of analyzing the main elements as well as heavy metals in just one digest and leads to comparable results. Furthermore, it is the same method as defined in the European Standard EN 16174 for the analysis of sludge, treated biowaste, and soil. Hence, for regulation purpose and for basic quality control, we recommend approving this method also. Nevertheless, in situations when absolute concentrations are essential, for example, for process-oriented studies, analyte mass flow calculations, or other research and material design purposes, we advise resorting to the EBC's reference or an equivalent method.

Heavy Metals (Cd, Cr, Cu, Ni, Pb, Zn). Heavy metals and metalloids that are originally present in the feedstock either could be volatilized during the pyrolysis process (e.g., $\mathrm{Cd}, \mathrm{Pb}$, $\mathrm{Hg}$, As) or will inevitably be concentrated in the resulting biochar. ${ }^{30,31}$ Undesired contamination may occur during processing, for example, by $\mathrm{Ni}$ release from reactor steel or $\mathrm{Zn}$ release during storage in nonadapted tin containers. With the exception of $\mathrm{Hg}$ and $\mathrm{Pb}$, and probably $\mathrm{Cd}$ and $\mathrm{Cr}$, the heavy metals considered here are micronutrients for plants or groups of plants. ${ }^{32}$ However, both essential and nonessential elements are potentially toxic so that threshold concentrations must be established for biochar certification. Table 1 shows the mean values resulting from the analyses of $\mathrm{BC} 1-\mathrm{BC} 3$, all of which were well below the IBI/EBC limits ${ }^{1,10}$ for $\mathrm{BC} 1$ and $\mathrm{BC} 2$. Exceedingly higher mean concentrations were observed for BC3 due to its origin (sewage sludge) and its high ash content. In general, $\mathrm{Cd}$ was the metal with the lowest concentration and $\mathrm{Zn}$ the one with the highest.

The analysis of trace metals in biochar is challenging because of the recalcitrance of the carbonaceous matrix to degradation and acid dissolution. Different decomposition techniques have been applied and compared. ${ }^{33}$ A preliminary interlaboratory exercise highlighted potential disagreements of the analytical results due to the different digestion procedures. ${ }^{31}$ In the case of wet digestion, different systems were used: $\mathrm{HNO}_{3} / \mathrm{HCl}$ ( $\left.\mathrm{LAB} \_06, \mathrm{LAB} \_08, \mathrm{LAB} \_24\right), \mathrm{HNO}_{3} / \mathrm{H}_{2} \mathrm{O}_{2}$ (LAB_05A, $\mathrm{LAB} \_\overline{1}$, $\mathrm{LAB} \_-\overline{1}$ ), and $\mathrm{HF} / \mathrm{HNO}_{3} / \mathrm{H}_{2} \mathrm{O}_{2}$ ( $\left.\mathrm{LAB} \_04\right)$. Ashing was adopted by LAB_11 and $\mathrm{LAB} \_21$, followed by acid treatment with $\mathrm{HCl}$ and $\mathrm{HNO}_{3} / \mathrm{H}_{2} \overline{\mathrm{O}}_{2}$, respectively. Instrumental analysis was conducted by ICP-MS, ICP-OES, GFAAS, and AAS. For details, see Table S1.

Almost $90 \%$ of the total possible determinations (i.e., number of participating laboratories times total number of heavy metals) were performed, and $90 \%$ of them produced values within the tolerance limit $(\mathrm{Zu}$ scores $<|3.0|)$. However, important differences were observed. $\mathrm{Zu}$ scores $>|3.0|$ were obtained once for $\mathrm{Cd}, \mathrm{Cu}$, and $\mathrm{Pb}$, but three, four, and six times for $\mathrm{Ni}, \mathrm{Cr}$, and $\mathrm{Zn}$, respectively (Figure 1). Both repeatability and reproducibility tended to decrease in the order $\mathrm{BC} 3>\mathrm{BC} 2>\mathrm{BC} 1$ (e.g., relative reproducibility $\mathrm{SD}$ : $\mathrm{BC} 3$ (from $23(\mathrm{~Pb})$ to $58 \%(\mathrm{Cd})$ ), $\mathrm{BC} 2$ (from $41(\mathrm{Cu})$ to $82 \%$ $(\mathrm{Cr})$ ), and $\mathrm{BC} 1$ (from $45(\mathrm{Zn})$ to $458 \%(\mathrm{Cd})$ ) (Table 1). This trend is probably due to the decreasing ash and increasing OM contents (i.e., higher matrix effect at lower metal concentrations). HorRat values ranged from 3.0 to 18.6 and were thus generally unsatisfactory. In the cases of $\mathrm{BC} 1$ and $\mathrm{BC} 2$, the Kernel density plots (Figure S2, various pages) generally presented bi- to multimodal probability density distribution, with most of the values clustered at the lower concentration mode where the reference value was positioned. An opposite situation was observed for $\mathrm{BC} 3$.

Six laboratories (LAB_04, LAB_05A, LAB_08, LAB_13, LAB_16, LAB_24) of $\overline{10}$ exhibited at least $90 \%$ of concentration values within the tolerance limit $(\mathrm{Zu}$ scores $<|3.0|)$. These laboratories used different spectroscopies (ICP-MS, GFAAS, AAS, ICP-OES; Table S1), suggesting that the instrumental approach was not a crucial factor for the accuracy 
at least for the given metals and the concentrations encountered here. As mentioned above, the different pretreatments could be the main cause of the observed disagreements. The use of $\mathrm{HNO}_{3}$ alone or with $\mathrm{H}_{2} \mathrm{O}_{2}, \mathrm{HF}$, or $\mathrm{HCl}$ (aqua regia) was common to all of the digestion procedures. Values at the lower tolerance limits were apparently found in samples subjected to ashing ( $L A B \_11$ and $L A B \_21$ ) in the case of BC3 (e.g., Cr, Figure $S 1, \mathrm{p}$ 88). Lower values could be tentatively associated with volatilization during thermal treatment for metals such as $\mathrm{Cd}, \mathrm{Zn}$, and $\mathrm{Pb} .^{30,31,34}$

A comparison of the mean value with the reference value (LAB_04) showed, in some cases, significant differences that increased from $\mathrm{BC} 1$ to $\mathrm{BC} 3$ with the concomitant increase of ash content (Table 1). However, on a relative basis these discrepancies slightly increased in the order BC3 (average deviations 18\%) < BC2 (26\%) < BC1 (33\%). The highest deviations were observed for $\mathrm{Cr}$ and $\mathrm{Zn}$, indicating that these metals may be the most problematic during biochar analysis. Whereas data from $\mathrm{BC} 1$ revealed both positive and negative discrepancies, for $\mathrm{BC} 3$, the mean values were always lower than the reference value for all analyzed metals. This fact may be related to the extraction with hydrofluoric acid (HF) that can result in leaching of additional metals from the decomposition of siliceous components. However, working with highly toxic HF could be avoided for biochars derived from feedstock with low silicon or low ash contents. It was demonstrated that microwave digestion with $\mathrm{HNO}_{3} / \mathrm{H}_{2} \mathrm{O}_{2}$ can provide good results for the determination of trace elements in coals, but $\mathrm{HF}$ was required to increase their recovery from ash-rich coal and coal ash. ${ }^{35,36}$ Operative conditions are crucial in microwave irradiation, and under severe conditions (closed vessels, high temperatures) the digestion can be performed satisfactorily with $\mathrm{HNO}_{3}$ alone (e.g., LAB_16). ${ }^{34}$

In summary, the determination of trace metals in a typical biochar (BC1) with $\mathrm{HNO}_{3}$ mixtures, with or without $\mathrm{HF}$, was adequate in combination with microwave digestion (concordance between mean and reference values, $\mathrm{Zu}$ scores, literature data from coals $\left.{ }^{28,29}\right)$. In the case of a typical ash-rich char (e.g., BC3), the use of HF may be required for wet digestion, whereas ashing was not as efficient as with low-ash biochar. No evidence of differences in the instrumental techniques (ICP-MS, ICP-OES, GFAAS) was found. The results depended on type of biochar and metal; typical biochar (BC1) exhibited the highest data variability (low reproducibility, multimodal distribution), probably due to the recalcitrant carbonaceous network, whereas the most difficult metals were $\mathrm{Cd}$ (the least abundant), $\mathrm{Cr}$, and $\mathrm{Zn}$.

Polycyclic Aromatic Hydrocarbons (PAH). Biochar usually contains $\mathrm{PAH}$ of a few, up to a few hundred, milligrams per kilogram (sum of the 16 U.S. EPA PAHs; PAH_Sum). ${ }^{37}$ All three biochars were at the lower end of this range $(843 \mu \mathrm{g} / \mathrm{kg}<$ mean value $<2893 \mu \mathrm{g} / \mathrm{kg}$, Table 1) and would pass current quality standards set by the EBC and the IBI. ${ }^{1,10}$ For further details regarding $\mathrm{PAH}$ formation during biochar production, as well as biochar quality and certification, see Bucheli et al. ${ }^{37}$

The $\mathrm{Zu}$ scores for PAH_Sum were $<|3.0|$ for all five participating laboratories, indicating that no major deviations in the reported data occurred (Figure 1). Correspondingly, all box plots were within the limits of tolerance (Figure S1). The reference value in relation to the other reported data was highest in $\mathrm{BC} 1$ (by almost a factor of 2 or more), as well as in BC2, and was second highest in BC3 (Figure S1, pp 21, 61, 101). The Kernel density plots (Figure S2, pp 141, 181, 221) of the PAH Sum show a rather normal probability density distribution for all three samples. Relative repeatability SD (6\%) was up to a factor of 10 better than relative reproducibility SD (40-67\%) (Table 1). Together with HorRat from 2.4 to 4.9, this indicates some systematic differences between results of individual participants. Note that some laboratories calculated PAH_Sum despite concentrations of some single analytes being below the limits of quantification. This led to sometimes higher numbers of laboratories and results for PAH Sum than for individual compounds.

Such methodological differences were suspected to limit $\mathrm{PAH}$ data comparison between laboratories already in an earlier study. ${ }^{37}$ Concentrations determined with toluene extraction, found to be best suited for biochar, ${ }^{38}$ distributed in most cases in the upper half of the box plots/Kernel density plots, whereas acetone/cyclohexane $(1: 1, \mathrm{v} / \mathrm{v})$ provided lower concentrations, particularly in $\mathrm{BC} 1$ (LAB_05A, LAB_05B). The situation was most pronounced in the case of acetonitrile (LAB_19; not included in the box plot and Kernel density plots of $\mathrm{PAH}$ Sum), which resulted in concentrations below the limits of quantitation $(<500 \mu \mathrm{g} / \mathrm{kg})$. A more detailed evaluation of methodological differences and their potential influences on reported concentrations remains difficult, mostly because of the limited number of participants.

The most dominating individual PAH in biochar is generally naphthalene (NAP), ${ }^{37}$ which was confirmed here by all three biochars $(40 \%<$ mean value $(\mathrm{NAP} / \mathrm{PAH}$ _Sum $)<46 \%$, Table 1). Simultaneously, it is one of the analytically most challenging ones, because of its pronounced volatility, which makes it prone to both losses and cross-contamination during extraction and extract concentration. Despite such difficulties, $\mathrm{Zu}$ scores were consistently $<|3.0|$ (Figure 1), and relative reproducibility SD values (25-84\%; Table 1) as well as HorRat (1.3-5.2, Table 1) were inconspicuous in comparison with other individual PAHs. Still, Kernel density plots (Figure S2, pp 155, 195, 235) varied from sample to sample and were normally (monomodally) distributed in $\mathrm{BC} 2$, bimodal in $\mathrm{BC} 3$, and trimodal in $\mathrm{BC} 1$, illustrating the analytical problems related to this compound. Again, the results of acetonitrile extraction were among the lowest values for all three samples, indicating the low suitability of this solvent for PAH extraction from biochar.

The next important $\mathrm{PAH}$ in terms of its relative contribution to the PAH Sum was phenanthrene (PHE) $(11 \%$ < mean (NAP/PAH_Sum $)<15 \%$, Table 1). All interlaboratory comparison parameters ( $\mathrm{Zu}$ scores (Figure 1), levels of tolerance (box plots, Figure S1), and Kernel density plots (Figure S2)) showed a normal behavior, apart from a slight right shoulder of the probability density for BC 3 (Figure S2, p 236).

Of the remaining PAHs, the analytically more challenging compounds generally exhibited higher variations, for example, light ones such as acenaphthene (ACE) or anthracene (ANT), those that may be more difficult to separate such as benzo[b]fluoranthene $(\mathrm{BBF})$ and benzo[ $k]$ fluoranthene $(\mathrm{BKF})$, or those with lower concentrations such as dibenzo $[a, h]$ anthracene (DBA). For instance, $\mathrm{Zu}$ scores $>|3.0|$ were observed for ACE (BC1, LAB_04; BC3, LAB_16), acenaphthylene (ACY) (BC3,

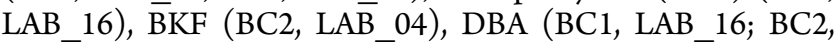
$\left.\mathrm{LAB}{ }_{-} 16 ; \mathrm{BC} 3, \mathrm{LAB} \_16\right)$, and fluorene (FLU) (BC3, LAB_16) (Figure 1). This is mirrored by the exceedance of tolerance levels and the non-normal Kernel density plots (see corresponding Figures S1 and S2). Multimodal probability density was additionally observed for benzo $[a]$ pyrene (BAP; BC2, $\mathrm{BC} 3)$, benzo[ghi]perylene (BPE; BC2), fluoranthene (FLA; 
$\mathrm{BC} 2, \mathrm{BC} 3$ ), indeno[1,2,3-cd]pyrene (IND; $\mathrm{BC} 1, \mathrm{BC} 3$ ), and pyrene (PYR; BC3) (Figure S2).

In summary, the amount of $\mathrm{PAH}$ data compiled and evaluated in this interlaboratory comparison is rather limited but supports general observations reported earlier regarding the quantification of these analytes in biochar (Bucheli et al., ${ }^{37}$ and references cited therein). The single most influencing parameter was repeatedly shown to be the extraction solvent, and it is generally agreed that toluene is the solvent of choice. ${ }^{38,39}$ Further analytical methodological recommendations are provided in Bucheli et al. ${ }^{37}$

Further Parameters. $p H$. Depending on feedstock and pyrolysis parameters, the $\mathrm{pH}$ of biochar may vary between slightly acidic and strongly alkaline. ${ }^{40}$ As the quasi-inert $\mathrm{C}$ lattice of the biochar is neutral, the $\mathrm{pH}$ of the biochar composite depends mostly on the form and quantity of mineral ashes, functional surface groups, soluble hydroxides, and carbonates, as well as pyrolytic condensates precipitated on biochar surfaces. Pyrolysis temperature determines the chemical form of the ashes that sinter at high temperatures, leading to higher $\mathrm{pH}$ values. It also determines the chemical constitution of condensates that are more acidic at lower temperatures and more strongly adsorbed at higher temperatures. ${ }^{40}$

Whereas $\mathrm{BC} 1$ and $\mathrm{BC} 2$ were alkaline (mean = 9.9 and 9.3, respectively), BC3 was close to neutral (mean $=7.1)($ Table 1$)$. Considering that pyrolysis temperatures were highest for $\mathrm{BC} 1$ and BC3 (620 and $600{ }^{\circ} \mathrm{C}$, respectively) and only slightly lower for $\mathrm{BC} 2\left(500^{\circ} \mathrm{C}\right)$, the differences are most probably due to the different feedstock. As the ash content was highest for BC3 (72.5\%), lowest for $\mathrm{BC} 1$ (11.0\%), and intermediate for $\mathrm{BC} 2$ (34.8\%) (Table 1), the chemical form of the ashes and the strength of association to the biochar matrix seem most determining for the $\mathrm{pH}$ of the respective biochars.

The $\mathrm{Zu}$ scores for $\mathrm{pH}$ were well below $|3.0|$ for all laboratories except $\mathrm{LAB} \_17$, indicating that no major deviations in the reported data occurred (Figure 1). The reference values for $\mathrm{BC} 1$ (8.3) and $\mathrm{BC} 2(8.3)$ were at the lower end in relation to the other reported values, whereas it coincided with the mean value (7.1) for BC3 (Figure S2, pp 39, 79, 119). The Kernel density plots (Figure S2, pp 159, 199, 239) showed a rather normal probability density distribution for $\mathrm{BC} 3$, whereas for $\mathrm{BC} 1$ and $\mathrm{BC} 2,84 \%$ of laboratories showed a normal probability density distribution in mode 3 (BC1, $9.7<\mathrm{pH}<10.7$; $\mathrm{BC} 2$, $9.1<\mathrm{pH}<9.8$ ) and $12 \%$ in mode 2 (BC1, $8.3<\mathrm{pH}<8.5$; $\mathrm{BC} 2,8.1<\mathrm{pH}<8.4)$. These two distinct density distributions for $\mathrm{BC} 1$ and $\mathrm{BC} 2$ point to a systematic difference of the analytical method used by the respective laboratories. The reference values of both $\mathrm{BC} 1$ and $\mathrm{BC} 2$ were positioned in the lower density distribution of mode 2 ( $12 \%$ of laboratories). The relative repeatability SD $(0.3-0.9 \%)$ was roughly a factor of 20 better than the relative reproducibility SD (4.5-6.6\%) (Table 1), confirming that rather methodological differences were responsible for the variations.

All laboratories contributing to the mode 3 density distribution of $\mathrm{BC} 1$ and $\mathrm{BC} 2$ and all those that plot highest in the box plots (Figure S1, pp 39, 79) measured the $\mathrm{pH}$ in a $\mathrm{H}_{2} \mathrm{O}$ suspension $\left(\mathrm{pH}\left(\mathrm{H}_{2} \mathrm{O}\right)\right)$, whereas the laboratories within mode 2 (12\% of laboratories) suspended their samples in a 0.01 $\mathrm{M} \mathrm{CaCl}_{2}$ solution. Within a given mode, the methodological differences originated mostly from the various amounts of solvent in use $(1: 2.5-1: 20(\mathrm{w} / \mathrm{v}))$. Further methodological differences comprised milling, drying, and sieving of the sample prior to analysis. As all $\mathrm{pH}\left(\mathrm{H}_{2} \mathrm{O}\right)$ analyses plotted, except two outliers, in a narrow band and showed a normal distribution, no systematic influence of these differences can be deduced. No consistent explanation was found for the outliers of $L A B \_18$ for BC1 and $L A B \_13$ for BC2. The outlier $L A B \_17 B$ for BC1, $\mathrm{BC} 2$, and $\mathrm{BC} 3$ might, however, be a consequence of not having milled or dried the samples and only having shaken the solution for $30 \mathrm{~min}$ (instead of 60 to $90 \mathrm{~min}$ as the others), although it is more likely that a wrong calibration or a defective $\mathrm{pH}$-measuring device caused such high differences compared to all other laboratories.

Four laboratories used $0.01 \mathrm{M} \mathrm{CaCl}_{2}$ instead of $\mathrm{H}_{2} \mathrm{O}$ for suspension, although with different mixing rates (from 1:2.5 to 1:10 (w/v)). Whereas the reference LAB 04, LAB 11, and $\mathrm{LAB} \_07 \mathrm{~A}$ delivered very similar results for all three chars, the values of $\mathrm{LAB}$ 17B were the lowest for $\mathrm{BC} 1$ and $\mathrm{BC} 2$ and the highest for $\mathrm{BC} 3$, probably for the same reasons as stated above. A suspension with $\mathrm{CaCl}_{2}(0.01 \mathrm{M})$ is commonly used for $\mathrm{pH}$ analysis of compost and soil, resulting in values that are generally $0.3-1.0 \mathrm{pH}$ unit lower than those obtained with $\mathrm{H}_{2} \mathrm{O}$ extraction. We assume that $\mathrm{CaCl}_{2}$ solutions extracted more strongly exchangeable protons from the highly porous and $\mathrm{C}$-rich biochars $\mathrm{BC} 1$ and $\mathrm{BC} 2$, yielding $\mathrm{pH}$ values that were lower by 0.9 to $1.05 \mathrm{pH}$ units compared to $\mathrm{pH}\left(\mathrm{H}_{2} \mathrm{O}\right)$. However, no significant difference between the extraction with $\mathrm{CaCl}_{2}$ and that with $\mathrm{H}_{2} \mathrm{O}$ was observed for the ash-rich biochar BC3, most likely because of the low $\mathrm{pH}$-buffering capacity of ash.

In summary, $\mathrm{pH}$ analyses in $\mathrm{H}_{2} \mathrm{O}$ or $\mathrm{CaCl}_{2}$ solutions both resulted in consistent values, although the use of $\mathrm{CaCl}_{2}$ solutions delivered lower $\mathrm{pH}$ values at lower ash contents. For the ease of comparability with results from the usual soil and compost method, it is recommended to determine the $\mathrm{pH}$ in $\mathrm{CaCl}_{2}$ solution. There are no clear methodological preferences concerning the dilution factor. Still, we propose to use $1: 10$ as a consensus: it might become difficult to yield a suspension with lower dilution factors because of the high water holding capacity of some biochars. It is further suggested to use dried samples (at $40{ }^{\circ} \mathrm{C}$ until constant weight), milling to a particle size $<2 \mathrm{~mm}$ (but no sieving), shaking for $1 \mathrm{~h}$, and measuring the $\mathrm{pH}$ in the aqueous phase after sedimentation of the solid phase.

Electrical Conductivity (EC). The so-called EC of biochar is a somewhat misleading technical term, as it does not deliver the EC of the material itself but of its dissolvable fraction in water. As biochar is, depending on the pyrolysis temperature and its feedstock, either an insulator, a semiconductor, or a conductor, the EC of the solid biochar itself is a very important characterizing parameter. ${ }^{41,42}$ However, the analytical method for biochar EC is adapted from measurements of the salt content of soil, compost, or other similar substrates. To avoid confusion, the EC of the biochar material itself is mostly given as electrical resistance. In the following, EC is considered as EC of the salts washed off from biochar. Hence, the EC depends mainly on the salt content, salt composition, and the salt's affinity to biochar.

The highest EC was determined for $\mathrm{BC} 1(1203 \mu \mathrm{S} / \mathrm{cm})$, followed by BC2 $(1054 \mu \mathrm{S} / \mathrm{cm})$ and BC3 $(785 \mu \mathrm{S} / \mathrm{cm}$ ) (Table 1 , mean values), which is the reverse of the order observed for the ash content of the samples. Note that $\mathrm{BC} 1$ contained the lowest amounts of $\mathrm{Ca}, \mathrm{K}$, and $\mathrm{Na}$, too. It is evident that, in this case, the EC was not only determined by the ash content but also depended on the alkalinity of the aqueous solution. In general, the EC is highly dependent on dissolvable $\mathrm{OH}$ moieties, as well as on dissolvable monovalent cations (in the case of biochar, mainly $\mathrm{K})$. 
The reference value of all three biochars was between 18 and $28 \%$ lower than the mean value (Table 1 ). The $\mathrm{Zu}$ score for EC was $<|3.0|$ for all laboratories (Figure 1), indicating that no major deviations in the reported data occurred.

The Kernel density plots showed a rather normal probability density distribution for all three $\mathrm{BC}$ with a shoulder and a second maximum around $2000 \mu \mathrm{S} / \mathrm{cm}$ for $\mathrm{BC} 1, \mathrm{BC} 2$, and $\mathrm{BC} 3$, respectively (Figure S2, pp 130, 170, 210). BC1 and BC2 exhibited a high normal probability density distribution in mode 1 (92 and 100\%, respectively), whereas BC3 plotted about a fourth of the EC values in mode 2 (24\%). This points to a systematic difference caused by the respective analytical method. The reference value for $\mathrm{BC} 1$ is at the apex of the mode 1 distribution curve, and at the lower part for $\mathrm{BC} 2$ and $\mathrm{BC} 3$. Whereas relative repeatability SD was reasonably low (1.5-2.6\%), relative reproducibility SD was considerably higher (68-70\%) (Table 1), indicating systematic differences between the applied methods.

All laboratories dispensed the sample in $\mathrm{H}_{2} \mathrm{O}$, but the dilution factors varied between 1:2.5 and 1:25 (w/v). Although the laboratory applying the lowest dilution factor of $1: 25$ (LAB_16) yielded consistently the lowest EC values for all three biochars (Figure S1, pp 10, 50, 90), no correlation between EC and lower dilution factors between 1:2.5 and 1:20 was observed. $\mathrm{LAB} \_06$, and LAB_18 consistently delivered the

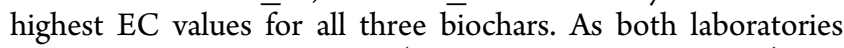
diluted with different factors (1:2.5 and 1:20, respectively), the best explanation for the elevated numbers may be that both laboratories did not filter the solution and measured EC in the slurry. As higher temperature biochars, such as BC1 and $\mathrm{BC} 3$, are good or at least partially good conductors and ion adsorbers, suspended biochar particles can affect the EC of the solution. Although not all laboratories working without filtering yielded higher EC values (e.g., $\mathrm{LAB} 21$ is rather at the lower end), all laboratories applying filtration prior to $E C$ measurements (LAB_04, LAB_05A, LAB_07A, LAB_08, LAB_24) obtained values that were close to the mean and gathered in mode 1 of the Kernel density distribution (Figure S2, pp 130, $170,210)$. Some biochars were milled and dried during sample preparation, whereas others where sieved and not dried or just used as received, but too few data are available to discern a consistent difference.

In summary, EC analysis with $\mathrm{H}_{2} \mathrm{O}$ as extracting agent can deliver consistent values. It is proposed to use dried samples that are crushed or milled to $<2 \mathrm{~mm}$. There are no clear methodological preferences concerning the dilution factor, although it is proposed to use $1: 10(\mathrm{w} / \mathrm{v})$ as consensus and to shake it for $1 \mathrm{~h}$ at $25^{\circ} \mathrm{C}$, followed by $5-7 \mu \mathrm{m}$ filtration. The result should refer to a $0.01 \mathrm{M} \mathrm{KCl}$ solution at $25^{\circ} \mathrm{C}$.

Specific Surface Area (SSA). The results reported by eight of the ring trial laboratories involved in this interlaboratory comparison (13 results per sample) were obtained with physical gas adsorption characterization, which is the most widely applied technique for total SSA (single- and multipoint BrunauerEmmett-Teller (BET) model) and pore size distribution characterization, being also the one recommended by the EBC and IBI. A summary of experimental conditions and results is presented in Table $S 1$ and Table 1, respectively.

The $\mathrm{Zu}$ scores for the SSA were $<|2.0|$ for samples $\mathrm{BC} 1$ and $\mathrm{BC} 2$ (Figure 1a,b) indicating no important deviation in the reported data. For $\mathrm{BC} 3$, the $\mathrm{Zu}$ scores were $<|2.0|$ for all laboratories except for LAB_04, with a value between $|2.0|$ and |3.0| (Figure 1c). Correspondingly, all of the box plots were within the limits of tolerance for all three samples (Figure S1, pp 42, 82, 122).

Looking at the Kernel density plots, $\mathrm{BC} 1$ and $\mathrm{BC} 2$ showed a normal probability density distribution (Figure S2, pp 162, 202), whereas BC3 showed a bimodal distribution (Figure S2, p 242), with the first mode corresponding to the value with the higher $\mathrm{Zu}$ score from LAB_04.

The relative repeatability SD ranged from 0.1 to $1.7 \%$ (Table 1), with the highest value for BC2. Although these results are very positive, they should not be taken as representative because only two laboratories provided more than one measurement. The relative reproducibility SD ranged from 19 to $29 \%$.

The highest SSA was observed for BC1 (with the best reproducibility), followed by $\mathrm{BC} 2$ (with the worst reproducibility) and BC3, with mean values around 320,100 , and $57 \mathrm{~m}^{2} / \mathrm{g}$, respectively (Table 1 ). This is in good agreement with the degree of thermal conversion of each sample; that is, higher pyrolysis temperature (shown for example with the higher $\mathrm{C}$ and lower $\mathrm{O}$ content) led to higher SSA. However, by comparison of some of the obtained values and applied methods given by each laboratory, some conclusions can be drawn regarding the influence of sample pretreatment, measurement conditions, and applied models on the total SSA determination.

Influence of Particle Size; Samples Milled or Nonmilled. LAB_01 and LAB_19 employed the same measurement conditions $\left(\mathrm{N}_{2}\right.$ adsorption at a partial pressure ratio of $0.05-$ $0.3 \mathrm{p} / \mathrm{p}^{0}$ ) with very similar degassing conditions (sample cleaning, in vacuum $2 \mathrm{~h}, 150{ }^{\circ} \mathrm{C}$, and $3 \mathrm{~h}, 100{ }^{\circ} \mathrm{C}$, respectively) but with different particle sizes, milled and nonmilled, respectively. The SSAs provided by LAB_19 were significantly lower than those from LAB 01 (Figure $S 1$, pp 42, 82, 122). Probably, this was due to lower pore accessibility of nonmilled samples, which was associated mostly with the "bottleneck" phenomena. This effect was significantly more pronounced for samples BC1 and BC2 (especially large SSAs in this sample) than for BC3. The results of $\mathrm{LAB}_{-} 04$ (providing the smallest SSAs for $\mathrm{BC} 1$ and $\mathrm{BC} 3$ ), where the samples were also not milled, support the conclusion that the particle size of the sample affects the measured SSA. However, other reasons cannot be excluded (see below).

On the basis of our results, it is recommended to mill the samples before SSA analysis to reduce diffusion limitations. At the best, the particle size should be reduced until the measured SSA remains constant. Practically, the recommendation is to mill the samples to a particle size $<1 \mathrm{~mm}$, although it could be argued that the determined SSA is then not representative for the biochar particles commonly applied to soil. However, the presence of diffusion limitations (no equilibrium) leads to biased results and is therefore nonrepresentative.

Influence of Degassing Conditions. Comparing the results from LAB_01 and $L A B \_17$, of which the main procedural difference consisted in the degassing step (either in vacuum or under $\mathrm{N}_{2}$ atmosphere, respectively), indicates that this sample pretreatment parameter may not considerably alter the final SSA value (Figure S1, pp 42, 82, 122). The effects of the applied maximum temperature and degassing time, if any, are also not clear. However, the maximum temperature should be low enough to avoid modifications of the structure of the biochar, which is in particular true under critical vacuum. Therefore, we recommend to start with a temperature between 100 and $200{ }^{\circ} \mathrm{C}$ and increase the treatment time until no change of SSA is detected. 
Influence of Adsorbate. In contrast to the other laboratories that used $\mathrm{N}_{2}, \mathrm{LAB} \_21$ worked with $\mathrm{CO}_{2}$ as adsorbate. It is well-known that $\mathrm{CO}_{2}$ is most appropriate to characterize small micropores, due to lower diffusion limitations, related to the measurement temperature ( 0 vs $\left.-196{ }^{\circ} \mathrm{C}\right)$. LAB_21 and LAB_01 performed similar sample pretreatment, which resulted in similar SSA for $\mathrm{BC} 1$ and $\mathrm{BC} 3$, but for $\mathrm{BC} 2$ significantly higher values were obtained by the use of $\mathrm{CO}_{2}$. Most probably, $\mathrm{BC} 2$ contained significantly more micropores and especially small micropores with low accessibility than samples BC1 and BC3. This is also in line with the higher impact of particle milling on SSA of BC2 compared to the two other samples and may explain the unsatisfactory reproducibility. The use of both $\mathrm{N}_{2}$ and $\mathrm{CO}_{2}$ as adsorbates would give complementary information on the pore structure.

Influence of Applied Method: Single- versus Multipoint BET Models. All laboratories applied the multipoint BET model with exception of LAB_04, which used the single-point BET model. The latter showed the smallest SSA of all measurements for BC1 and BC3. This may be explained with the applied BET model, together with the fact that here the samples were not milled. The single-point BET model is more appropriate for samples with a low variation in pore size. However, for samples as complex as pyrolysis chars, in which micro-, meso-, and macropores coexist, the application of this method may lead to over- or underestimation of the total SSA, depending at which partial pressure ratio the measurement is performed and which type of pores are dominating in the sample.

Consequently, it is recommended to measure several adsorption points over a wider partial pressure ratio $\left(0.005-0.3 \mathrm{p} / \mathrm{p}^{0}\right)$ and select the region of the isotherm that fits the mathematical model of BET best. It must be taken into account that this will change for each char sample. A better understanding of the porous structure of the sample would be obtained if several models were applied on the complete adsorption and desorption isotherm.

\section{SUMMARY AND RECOMMENDATIONS}

The first objective of this study was to assess the reliability of methods used for biochar analysis by organizing an interlaboratory comparison with free choice of analytical methods. As the need to analyze a broad spectrum of parameters in biochar on a larger scale is quite recent, most of the participating laboratories did not apply analytical methods specific for biochar analysis. Instead, they used their standard methods that originally were developed for the analysis of organic wastes, soils, fertilizers, or coals. Therefore, it is not surprising that intralaboratory repeatability was generally good or at least acceptable, whereas interlaboratory reproducibility was mostly not. Apart from potentially inapt analytical methods, diverging sample preparation steps may have contributed to this low performance. Only 2 of 38 parameters featured a robust mean that indicated a good comparability of values, namely, $\mathrm{C}$ and $\mathrm{pH}$ with a mean reproducibility $\mathrm{SD}<10 \%$ (Table 1 ). For $\mathrm{H}$, ash, and $\mathrm{P}$ the mean reproducibility $\mathrm{SD}$ values were between 10 and $20 \%$, which may be considered acceptable for this type of study. The variability of all other parameters was too high to be reliable, showing the urgent need to improve and standardize methods for biochar analysis. Specific methodological recommendations for biochar analysis of individual parameters were given. Nevertheless, depending on the purpose of a given investigation, its regulatory context, and the analytical methods at hand, biochar researchers may still resort to alternative methods, using the present interlaboratory comparison as a base to properly evaluate and discuss their results. Future quality assurance and quality control measures in biochar analysis should include the generation of a set of representative biochar reference materials and true round-robin tests with laboratories using biochar reference methods.

\section{ASSOCIATED CONTENT}

\section{Supporting Information}

The Supporting Information is available free of charge on the ACS Publications website at DOI: 10.1021/acs.jafc.5b05055.

Table S1 and Figures S1 and S2 (PDF)

\section{AUTHOR INFORMATION}

\section{Corresponding Authors}

*(T.D.B.) E-mail: thomas.bucheli@agroscope.admin.ch. Phone: +41 5846873 42. Fax: +41 584687201 .

*(H.-P.S.) E-mail: schmidt@ithaka-institut.org. Phone: +41 27 3981292.

\section{Funding}

We thank the European Cooperation in Science and Technology (COST) for financial support.

Notes

The authors declare no competing financial interest.

\section{ACKNOWLEDGMENTS}

The biochar-producing companies Pyreg GmbH, Sonnenerde $\mathrm{GmbH}$, and Swiss Biochar $\mathrm{GmbH}$ are acknowledged for sample donation. We are grateful to the IBI and the EBC for fruitful discussions about analytical method standardization. D.F., A.B., and A.G.R. thank the Università di Bologna and Regione Emilia Romagna (Italy) APQ Ricerca Intervento a "Sostegno dello sviluppo dei Laboratori di ricerca nei campi della nautica e dell'energia per il Tecnopolo di Ravenna" and Denis Zannoni for laboratory assistance. F.Z. thanks the laboratory staff at the Institute of Soil Research, University of Natural Resources and Life Sciences (BOKU), Vienna, Austria.

\section{REFERENCES}

(1) European Biochar Certificate - Guidelines for a Sustainable Production of Biochar. European Biochar Foundation (EBC), Arbaz, Switzerland; http://www.european-biochar.org/biochar/media/doc/ ebc-guidelines.pdf (Version 6.1 of June 19th, 2015). DOI: 10.13140/RG.2.1.4658.7043 (accessed: September 10, 2015).

(2) Camps-Arbestain, M.; Amonette, J. E.; Singh, B.; Wang, T.; Schmidt, H. P. A biochar classification system and associated test methods. In Biochar for Environmental Management. Science, Technology and Implementation; Lehmann, J., Joseph, S., Eds.; earthscan from Routledge: London, UK, 2015; pp 165-193.

(3) Goodman, A. L.; Busch, A.; Duffy, G. J.; Fitzgerald, J. E.; Gasem, K. A. M.; Gensterblum, Y.; Krooss, B. M.; Levy, J.; Ozdemir, E.; Pan, Z.; Robinson, R. L.; Schroeder, K.; Sudibandriyo, M.; White, C. M. An inter-laboratory comparison of $\mathrm{CO}_{2}$ isotherms measured on argonne premium coal samples. Energy Fuels 2004, 18, 1175-1182.

(4) von Lehmden, D. J.; Jungers, R. H.; Lee, R. E., Jr. Determination of trace elements in coal, fly ash, fuel oil, and gasoline - a preliminary comparison of selected analytical techniques. Anal. Chem. 1974, 46, 239-245.

(5) Birch, M. E. Analysis of carbonaceous aerosols: interlaboratory comparison. Analyst 1998, 123, 851-857.

(6) Watson, J. G.; Chow, J. C.; Chen, L. W. A. Summary of organic and elemental carbon/black carbon analysis methods and intercomparisons. Aerosol Air Qual. Res. 2005, 5, 65-102.

(7) Dembicki, H., Jr. An interlaboratory comparison of source rock data. Geochim. Cosmochim. Acta 1984, 48, 2641-2649. 
(8) EBC. Analytical Methods, version 4.1 (March 4, 2014); http:// www.european-biochar.org/en/analytical\%20methods (accessed Sept 10, 2015).

(9) Bucheli, T. D.; Bachmann, H. J.; Blum, F.; Buerge, D.; Giger, R.; Hilber, I.; Keita, J.; Leifeld, J.; Schmidt, H. P. On the heterogeneity of biochar and consequences for its representative sampling. J. Anal. Appl. Pyrolysis 2014, 107, 25-30.

(10) IBI (International Biochar Initiative). Standardized Product Definition and Product Testing Guidelines for Biochar That Is Used in Soil (IBI-STD-01.1, April 11, 2013); http://www.biochar-international. org/sites/default/files/IBI_Biochar_Standards_V1.1.pdf; 48 pp (accessed Sept 10, 2015).

(11) DIN 38402 A45:2014. German standard methods for the examination of water, waste water and sludge - General information (group A) - Part 45: Interlaboratory comparisons for proficiency testing of laboratories.

(12) Hampel, F. R.; Ronchetti, E. M.; Rousseeuw, P. J.; Stahel, W. A. Robust Statistics: The Approach Based on Influence Functions; Wiley: New York, 1986.

(13) Rousseeuw, P. J.; Croux, C. Alternatives to the median absolute deviation. J. Am. Stat. Assoc. 1993, 88, 1273-1283.

(14) Horwitz, W. Evaluation of analytical methods used for regulation of foods and drugs. Anal. Chem. 1982, 54, 67A-76A.

(15) Horwitz, W.; Albert, R. The Horwitz ratio (HorRat): a useful index of method performance with respect to precision. J. AOAC Int. 2006, 89, 1095-1109.

(16) Zhao, L.; Cao, X.; Masek, O.; Zimmerman, A. Heterogeneity of biochar properties as a function of feedstock sources and production temperatures. J. Hazard. Mater. 2013, 256, 1-9.

(17) Almendros, G.; González-Vila, F. J.; Martín, F. Fire-induced transformation of soil organic matter from an oak forest. An experimental approach to the effects of fire on humic substances. Soil Sci. 1990, 149, 158-168.

(18) Wang, T.; Camps-Arbestain, M.; Hedley, M. Predicting C aromaticity of biochars based on their elemental composition. Org. Geochem. 2013, 62, 1-6.

(19) Masiello, C. A. New directions in black carbon organic geochemistry. Mar. Chem. 2004, 92, 201-213.

(20) Schimmelpfennig, S.; Glaser, B. One step forward toward characterization: some important material properties to distinguish biochars. J. Environ. Qual. 2012, 41, 1001-1013.

(21) Bagreev, A.; Bandosz, T. J.; Locke, D. C. Pore structure and surface chemistry of adsorbents obtained by pyrolysis of sewage sludge-derived fertilizer. Carbon 2001, 39, 1971-1979.

(22) Knicker, H. "Black nitrogen" - an important fraction in determining the recalcitrance of charcoal. Org. Geochem. 2010, 41, 947-950.

(23) Cheah, S.; Malone, S. C.; Feik, C. J. Speciation of sulfur in biochar produced from pyrolysis and gasification of oak and corn stover. Environ. Sci. Technol. 2014, 48, 8474-8480.

(24) Fox, A.; Kwapinski, W.; Griffiths, B. S.; Schmalenberger, A. The role of sulfur- and phosphorus-mobilizing bacteria in biochar-induced growth promotion of Lolium perenne. FEMS Microbiol. Ecol. 2014, 90, $78-91$.

(25) Heiri, O.; Lotter, A. F.; Lemcke, G. Loss on ignition as a method for estimating organic and carbonate content in sediments: reproducibility and comparability of results. J. Paleolimnol. 2001, 25, 101-110.

(26) Daniel, R. C.; Lischer, P.; Theiller, G. Four new CII reference materials for the chemical analysis of plants: Pine needles, oak leaves, barley-straw and apple-fruit. In Developments in Plant and Soil Sciences, Optimization of Plant Nutrition; Fragoso, M. A. C., Van Beusichem, M. L., Eds.; Kluwer Academic Publishers: 1993; Vol. 53, pp 31-35.

(27) Davidson, C. M. Methods for the determination of heavy metals and metalloids in soils. In Heavy Metals in Soils. Trace Metals and Metalloids in Soils and Their Bioavailability, 3rd ed.; Alloway, B. J., Ed.; Springer: Dordrecht, The Netherlands, 2013; pp 97-140.

(28) Agrafioti, E.; Kalderis, D.; Diamadopoulos, E. Ca and $\mathrm{Fe}$ modified biochars as adsorbents of arsenic and chromium in aqueous solutions. J. Environ. Manage. 2014, 146, 444-450.
(29) Chen, B.; Chen, Z.; Lv, S. A novel magnetic biochar efficiently sorbs organic pollutants and phosphate. Bioresour. Technol. 2011, 102, 716-723.

(30) Evangelou, M. W. H.; Brem, A.; Ugolini, F.; Abiven, S.; Schulin, R. Soil application of biochar produced from biomass grown on trace element contaminated land. J. Environ. Manage. 2014, 146, 100-106.

(31) Van Wesenbeeck, S.; Prins, W.; Ronsse, F.; Antal, M. J., Jr. Sewage sludge carbonization for biochar applications. Fate of heavy metals. Energy Fuels 2014, 28, 5318-5326.

(32) Kabata-Pendias, A. Trace Elements in Soils and Plants, 4th ed.; CRC Press, Taylor and Francis Group, 2011.

(33) Enders, A.; Lehmann, J. Comparison of wet-digestion and dryashing methods for total elemental analysis of biochar. Commun. Soil Sci. Plant Anal. 2012, 43, 1042-1052.

(34) Wang, J.; Nakazato, T.; Sakanishi, K; Yamada, O.; Tao, H.; Saito, I. Single-step microwave digestion with $\mathrm{HNO}_{3}$ alone for determination of trace elements in coal by ICP spectrometry. Talanta 2006, 68, 1584-1590.

(35) Low, F.; Zhang, L. Microwave digestion for the quantification of inorganic elements in coal and coal ash using ICP-OES. Talanta 2012, $101,346-352$.

(36) Wang, J.; Nakazato, T.; Sakanishi, K.; Yamada, O.; Tao, H.; Saito, I. Microwave digestion with $\mathrm{HNO} 3 / \mathrm{H} 2 \mathrm{O} 2$ mixtures at high temperatures for determination of trace elements in coal by ICP-OES and ICP-MS. Anal. Chim. Acta 2004, 514, 115-124.

(37) Bucheli, T. D.; Hilber, I.; Schmidt, H. P. Polycyclic aromatic hydrocarbons and polychlorinated aromatic compounds in biochar. In Biochar for Environmental Management. Science, Technology and Implementation, 2nd ed.; Lehmann, J., Joseph, S., Eds.; earthscan from Routledge: London, UK, 2015; pp 595-624.

(38) Hilber, I.; Blum, F.; Leifeld, J.; Schmidt, H. P.; Bucheli, T. D. Quantitative determination of PAHs in biochar: a prerequisite to ensure its quality and safe application. J. Agric. Food Chem. 2012, 60, 3042-3050.

(39) Hale, S. E.; Lehmann, J.; Rutherford, D.; Zimmerman, A. R.; Bachmann, R. T.; Shitumbanuma, V.; O’Toole, A.; Sundqvist, K. L.; Arp, H. P. H.; Cornelissen, G. Quantifying the total and bioavailable polycyclic aromatic hydrocarbons and dioxins in biochars. Environ. Sci. Technol. 2012, 46, 2830-2838.

(40) DeLuca, T. H.; Gundale, M. J.; MacKenzie, M. D.; Jones, D. L. Biochar effects on soil nutrient transformations. In Biochar for Environmental Management. Science, Technology, and Implementation; Lehmann, J., Joseph, S., Eds.; earthscan from Routledge: London, UK, 2015; pp 421-454.

(41) Asada, T.; Ishihara, S.; Yamane, T.; Toba, A.; Yamada, A.; Oikawa, K. Science of bamboo charcoal: study on carbonizing temperature of bamboo charcoal and removal capability of harmful gases. J. Health Sci. 2002, 48, 473-479.

(42) Nishimiya, K.; Hata, T.; Imamura, Y.; Ishihara, S. Analysis of chemical structure of wood charcoal by X-ray photoelectron spectroscopy. J. Wood Sci. 1998, 44, 56-61. 